\title{
TWISTED LOGARITHMIC MODULES OF VERTEX ALGEBRAS
}

\author{
BOJKO BAKALOV
}

\begin{abstract}
Motivated by logarithmic conformal field theory and GromovWitten theory, we introduce a notion of a twisted module of a vertex algebra under an arbitrary (not necessarily semisimple) automorphism. Its main feature is that the twisted fields involve the logarithm of the formal variable. We develop the theory of such twisted modules and, in particular, derive a Borcherds identity and commutator formula for them. We investigate in detail the examples of affine and Heisenberg vertex algebras.
\end{abstract}

\section{INTRODUCTION}

The vertex operator realizations of affine Kac-Moody algebras LW, FK, KKLW, KP led to the introduction of the notions of a vertex algebra $\mathrm{B}$ and its twisted modules [Le, FLM, FFR, D. Twisted modules played an important role in the Frenkel-Lepowsky-Meurman construction of a vertex algebra with a natural action of the Monster on it [FLM]. Vertex algebras provide a rigorous algebraic description of two-dimensional chiral conformal field theory (see e.g. [BPZ, Go, DMS]), and twisted modules are important for studying orbifolds (see e.g. DHVW, DVVV, KT] among many other works).

Motivated by an example from logarithmic conformal field theory (see e.g. $[\mathrm{AM}, \mathrm{CR}]$ ), Y.-Z. Huang introduced in $[\mathrm{H}]$ a more general notion of a twisted module, for which the corresponding automorphism may have an infinite order and is not necessarily semisimple. The main feature of such twisted modules is that the twisted fields involve the logarithm of the formal variable. However, they lacked a Borcherds identity, $n$-th product identity, or commutator formula, all of which are powerful tools in the theory of vertex algebras. The difficulty was partly caused by the fact that the definition of $n$-th product of fields from [Li1, Li2] is not very convenient in the case of twisted modules. This problem was solved in [BM], where we showed that another formula for the $n$-th product $[\mathrm{BN}, \mathrm{BK} 2]$ remains valid in the twisted case.

Date: April 24, 2015.

2010 Mathematics Subject Classification. 17B69, 81R10.

Key words and phrases. Affine Kac-Moody algebra; Heisenberg algebra; twisted module; vertex algebra; Virasoro algebra. 
In the present paper, we use the formula from [BM] to provide another definition of a twisted module, more general than the one from $[\mathrm{H}]$. Our definition is in the spirit of [Li1, Li2, LL, so that the state-field correspondence map $Y$ is a homomorphism of vertex algebras relative to all $n$-th products. We develop a framework that allows many results about vertex algebras to be transferred to general twisted modules. In particular, we define a mode expansion of twisted fields and a shifted delta function. Our main results are a Borcherds identity and a commutator formula for general twisted modules. We investigate in detail the examples of affine and Heisenberg vertex algebras, and we plan to consider additional examples in the future. The theory developed here will be used in our joint work with T. Milanov, which aims to understand and utilize the vertex operators arising in Gromov-Witten theory (see [DZ1, DZ2, M, MT, FGM, BM, CV, LYZ, MST]).

Here is an outline of the present paper. In Section 2, we briefly review the basic definitions and properties of vertex algebras and their modules. This section can be skipped by readers familiar with the theory.

In Section 3, we introduce the notions of a logarithmic field, locality, and $n$-th products of logarithmic fields. We express the $n$-th product in terms of the normally ordered product and the propagator, and we prove that any local collection of logarithmic fields generates a vertex algebra.

In Section 4, we introduce the main object of the paper, the notion of a $\varphi$-twisted $V$-module where $\varphi$ is an arbitrary (not necessarily semisimple) automorphism of a vertex algebra $V$. When $\varphi$ is locally finite, we express it as $\varphi=\sigma e^{-2 \pi \mathrm{i} \mathcal{N}}$, where $\sigma \in \operatorname{Aut}(V)$ is semisimple and $\mathcal{N} \in \operatorname{Der}(V)$ is locally nilpotent.

Section 5 contains our main result, the Borcherds identity for $\varphi$-twisted modules. In particular, as a consequence, we derive a commutator formula for the logarithmic fields in a twisted module. We prove that the Borcherds identity can replace the locality and $n$-th product identity in the definition of a twisted module.

In Section 6, we describe all twisted modules of affine and Heisenberg vertex algebras in terms of modules over certain twisted versions of the corresponding Lie algebras. We also determine the action of the Virasoro algebra. For the Heisenberg vertex algebra, all twisted irreducible highestweight modules are constructed explicitly.

Throughout the paper, $z, z_{1}, z_{2}, \ldots$ will be commuting formal variables, and we will use the notation $z_{i j}=z_{i}-z_{j}$ and $x^{(k)}=x^{k} / k !$. All vector spaces will be over $\mathbb{C}$. We denote by $\mathbb{Z}_{+}$the set of non-negative integers.

\section{Preliminaries on Vertex Algebras}

In this section, we briefly review the basic definitions and properties of vertex algebras and their modules. For more details, we refer to [FLM, K2, FB, LL, KRR. 
2.1. Quantum fields. A (quantum) field on a vector space $V$ is a linear map from $V$ to the space of Laurent series $V((z))=V[[z]]\left[z^{-1}\right]$. The space of all fields

$$
\operatorname{Fie}(V)=\operatorname{Hom}_{\mathbb{C}}(V, V((z)))
$$

is closed under the derivative $\partial_{z}$. The composition $a(z) b(z)$ of two fields is not well defined in general. Instead, one considers the composition $a\left(z_{1}\right) b\left(z_{2}\right)$, which is a map from $V$ to $V\left(\left(z_{1}\right)\right)\left(\left(z_{2}\right)\right)$. Note that $V\left(\left(z_{1}\right)\right)\left(\left(z_{2}\right)\right)$ and $V\left(\left(z_{2}\right)\right)\left(\left(z_{1}\right)\right)$ are two different subspaces of $V\left[\left[z_{1}^{ \pm 1}, z_{2}^{ \pm 1}\right]\right]$ whose intersection is $V\left(\left(z_{1}, z_{2}\right)\right)=$ $V\left[\left[z_{1}, z_{2}\right]\right]\left[z_{1}^{-1}, z_{2}^{-1}\right]$.

A pair of fields $a, b$ is called local [Go, DL, Li1] if

$$
z_{12}^{N} a\left(z_{1}\right) b\left(z_{2}\right)=z_{12}^{N} b\left(z_{2}\right) a\left(z_{1}\right), \quad z_{12}=z_{1}-z_{2},
$$

for some integer $N \geq 0$. When applied to any $v \in V$, both sides of this equation become elements of $V\left(\left(z_{1}, z_{2}\right)\right)$. For $n \in \mathbb{Z}$, the $n$-th product $a_{(n)} b$ of two local fields $a, b$ is defined by (cf. [BN, BK2]):

$$
\left(a_{(n)} b\right)(z) v=\left.\partial_{z_{1}}^{(N-1-n)}\left(z_{12}^{N} a\left(z_{1}\right) b\left(z_{2}\right) v\right)\right|_{z_{1}=z_{2}=z}
$$

for $v \in V, n \leq N-1$, and $a_{(n)} b=0$ for $n \geq N$. It is easy to show that this definition is equivalent to the one due to H. Li Li1 (see KRR, Lecture 14]). Note that $a_{(n)} b$ is again a field and is independent of the choice of $N$ satisfying (2.1). Moreover, if $c$ is another field local with $a$ and $b$, then $c$ is local with $a_{(n)} b$ (Dong's Lemma [Li1, K2]; see Lemma 3.4 below).

The constant field $I$ equal to the identity operator is local with any other field $a$, and satisfies

$$
a_{(n)} I=0, \quad a_{(-n-1)} I=\partial_{z}^{(n)} a, \quad n \geq 0 .
$$

Let $\mathcal{V} \subset \operatorname{Fie}(V)$ be a local collection of fields, i.e., such that every pair $a, b \in \mathcal{V}$ is local. We will assume that $I \in \mathcal{V}$. By Dong's Lemma, the smallest subspace $\overline{\mathcal{V}} \subset \operatorname{Fie}(V)$ containing $\mathcal{V}$ and closed under all $n$-th products is again a local collection. Then $\overline{\mathcal{V}}$ is also closed under $\partial_{z}$.

2.2. Vertex algebras. A vertex algebra is a vector space $V$ (space of states), with a distinguished vector $\mathbf{1} \in V$ (vacuum vector) and a linear map $Y: V \rightarrow$ $\operatorname{Fie}(V)$ (state-field correspondence), such that $Y(\mathbf{1})=I$ and $Y(V)$ is a local collection of fields. The fields $Y(a)(a \in V)$ are usually written as

$$
Y(a, z)=\sum_{n \in \mathbb{Z}} a_{(n)} z^{-n-1}, \quad a_{(n)} \in \operatorname{End}(V),
$$

and the coefficients $a_{(n)}$ are called the modes of $a$. This endows $V$ with products $a_{(n)} b \in V$ for all $a, b \in V, n \in \mathbb{Z}$, and the map $Y$ is a homomorphism for all of them:

$$
Y\left(a_{(n)} b, z\right)=Y(a, z)_{(n)} Y(b, z) .
$$

The translation operator $T \in \operatorname{End}(V)$ is defined by $T a=a_{(-2)} \mathbf{1}$. Then

$$
[T, Y(a, z)]=\partial_{z} Y(a, z), \quad a \in V .
$$


A field $a(z)$ with this property is called translation covariant. By the Kac Existence Theorem [K2, DK, every local collection $\mathcal{V} \subset \operatorname{Fie}(V)$ of translation covariant fields generates a vertex algebra structure on $V$, provided that $V$ is linearly spanned by $\mathbf{1}$ and all coefficients of

$$
a_{1}\left(z_{1}\right) \cdots a_{r}\left(z_{r}\right) \mathbf{1}, \quad r \geq 1, a_{i} \in \mathcal{V} .
$$

Note that $\overline{\mathcal{V}}$, as defined above, is also a vertex algebra and the map $Y: V \rightarrow$ $\overline{\mathcal{V}}$ is an isomorphism [Li1, K2, DK].

For future use, recall that a derivation of $V$ is a linear operator $\mathcal{D}$ on $V$ such that

$$
\mathcal{D}\left(a_{(n)} b\right)=(\mathcal{D} a)_{(n)} b+a_{(n)}(\mathcal{D} b), \quad a, b \in V, \quad n \in \mathbb{Z} .
$$

The space $\operatorname{Der}(V)$ of all derivations is a Lie algebra containing $T$.

2.3. Borcherds identity. The main identity satisfied by the modes is the Borcherds identity (also called Jacobi identity [FLM]):

$$
\begin{gathered}
\sum_{i=0}^{\infty}(-1)^{i}\left(\begin{array}{c}
n \\
i
\end{array}\right)\left(a_{(m+n-i)}\left(b_{(k+i)} c\right)-(-1)^{n} b_{(k+n-i)}\left(a_{(m+i)} c\right)\right) \\
=\sum_{j=0}^{\infty}\left(\begin{array}{c}
m \\
j
\end{array}\right)\left(a_{(n+j)} b\right)_{(m+k-j)} c,
\end{gathered}
$$

where $a, b, c \in V$. Observe that the above sums are finite, because $a_{(j)} b=0$ for sufficiently large $j$. In particular, setting $n=0$ in the Borcherds identity, we obtain the commutator formula

$$
\left[a_{(m)}, b_{(k)}\right]=\sum_{j=0}^{\infty}\left(\begin{array}{c}
m \\
j
\end{array}\right)\left(a_{(j)} b\right)_{(m+k-j)} c .
$$

Equivalently,

$$
\left[Y\left(a, z_{1}\right), Y\left(b, z_{2}\right)\right]=\sum_{j=0}^{\infty} Y\left(a_{(j)} b, z_{2}\right) \partial_{z_{2}}^{(j)} \delta\left(z_{1}, z_{2}\right),
$$

where

$$
\delta\left(z_{1}, z_{2}\right)=\sum_{m \in \mathbb{Z}} z_{1}^{-m-1} z_{2}^{m}
$$

is the formal delta function. It is often convenient to use the formal expansions

$$
\begin{aligned}
& \iota_{z_{1}, z_{2}} z_{12}^{n}=\sum_{i=0}^{\infty}\left(\begin{array}{l}
n \\
i
\end{array}\right)(-1)^{i} z_{1}^{n-i} z_{2}^{i}, \\
& \iota_{z_{2}, z_{1}} z_{12}^{n}=\sum_{i=0}^{\infty}\left(\begin{array}{c}
n \\
i
\end{array}\right)(-1)^{n+i} z_{1}^{i} z_{2}^{n-i} .
\end{aligned}
$$

Then

$$
\partial_{z_{2}}^{(j)} \delta\left(z_{1}, z_{2}\right)=\left(\iota_{z_{1}, z_{2}}-\iota_{z_{2}, z_{1}}\right) z_{12}^{-j-1}, \quad j \geq 0 .
$$


The delta function has the property

$$
\operatorname{Res}_{z_{1}} a\left(z_{1}\right) \partial_{z_{2}}^{(j)} \delta\left(z_{1}, z_{2}\right)=\partial_{z_{2}}^{(j)} a\left(z_{2}\right)
$$

for any field $a(z)$, where as usual $\operatorname{Res}_{z}$ denotes the coefficient of $z^{-1}$.

2.4. Twisted modules. A representation (or module) of $V$ is a vector space $W$ endowed with a linear map $Y: V \rightarrow \operatorname{Fie}(W)$ such that $Y(\mathbf{1})=I$ and the Borcherds identity (2.3) holds for $a, b \in V, c \in W$ (see [FB, LL, KRR]). Equivalently, due to [Li1, one can replace (2.3) by the condition that $Y(V) \subset \operatorname{Fie}(W)$ is a local collection of fields satisfying the $n$-th product identity (2.2). The commutator formulas (2.4), (2.5) hold for modules as well.

Recall that an automorphism of a vertex algebra $V$ is an invertible linear operator $\sigma$ on $V$ such that

$$
\sigma\left(a_{(n)} b\right)=(\sigma a)_{(n)}(\sigma b), \quad a, b \in V, \quad n \in \mathbb{Z} .
$$

The group of all automorphisms of $V$ is denoted $\operatorname{Aut}(V)$. If $\sigma \in \operatorname{Aut}(V)$ has a finite order $r$, then $\sigma$ is semisimple with eigenvalues $r$-th roots of 1 . In the definition of a $\sigma$-twisted representation $W$ of $V$, the image of the above map $Y$ is allowed to have non-integral (rational) powers of $z$ (see [FFR, D, KRR] ). More precisely,

$$
Y(a, z)=\sum_{n \in p+\mathbb{Z}} a_{(n)} z^{-n-1}, \quad \text { if } \quad \sigma a=e^{-2 \pi \mathrm{i} p} a, \quad p \in \frac{1}{r} \mathbb{Z},
$$

where $a_{(n)} \in \operatorname{End}(W)$. Equivalently, the monodromy around $z=0$ is given by the action of $\sigma$ :

$$
Y(\sigma a, z)=Y\left(a, e^{2 \pi \mathrm{i}} z\right), \quad a \in V .
$$

The Borcherds identity (2.3) satisfied by the modes remains the same in the twisted case, provided that

$$
\sigma a=e^{-2 \pi \mathrm{i} m} a, \quad \sigma b=e^{-2 \pi \mathrm{i} k} b, \quad m, k \in \mathbb{Q}, \quad n \in \mathbb{Z} .
$$

As a consequence, we also have the commutator formula (2.4). However, (2.5) needs to be modified for twisted modules (see, e.g., BK1 and (5.10) below). It was proved in $\mathrm{BM}$ that in the definition of a twisted module the Borcherds identity can be replaced by the locality of all $Y(a, z)$ and the $n$-th product identity (2.2). Note that in the twisted case our definition of $n$-th product differs from H. Li's one from [Li1, Li2].

\section{LOGARITHMIC QUANTUM FIELDS}

In this section, we introduce the notions of a logarithmic field, locality, and $n$-th products of logarithmic fields. We express the $n$-th product in terms of the normally ordered product and propagator. We prove that any local collection of logarithmic fields generates a vertex algebra. 
3.1. Logarithmic fields and locality. As before, $z, z_{1}, z_{2}, \ldots$ will be formal variables, and let $\zeta, \zeta_{1}, \zeta_{2}, \ldots$ be another set of formal variables corresponding to them, which will be thought of as $\zeta=\log z$ and $\zeta_{i}=\log z_{i}$. More precisely, instead of $\partial_{z}$ and $\partial_{\zeta}$, we will work with the derivations

$$
D_{z}=\partial_{z}+z^{-1} \partial_{\zeta}, \quad D_{\zeta}=z \partial_{z}+\partial_{\zeta},
$$

and similarly for $D_{z_{i}}, D_{\zeta_{i}}$.

Fix a vector space $W$ over $\mathbb{C}$. For $\alpha \in \mathbb{C} / \mathbb{Z}$, we denote by $W[\zeta][[z]] z^{-\alpha}$ the space of all formal series of the form (cf. [BK2]):

$$
\sum_{i=0}^{\infty} w_{i}(\zeta) z^{i-m}, \quad w_{i}(\zeta) \in W[\zeta], \quad m \in \alpha .
$$

For example, $W[\zeta][[z]] z^{\mathbb{Z}}=W[\zeta]((z))$ is the space of Laurent series in $z$ with coefficients in $W[\zeta]$. Observe that $W[\zeta][[z]] z^{-\alpha}$ is a module over the ring $\mathbb{C}((z))$, and is closed under the derivations $D_{z}$ and $D_{\zeta}$.

Definition 3.1. With the above notation, let

$$
\operatorname{LFie}_{\alpha}(W)=\operatorname{Hom}_{\mathbb{C}}\left(W, W[\zeta][[z]] z^{-\alpha}\right), \quad \alpha \in \mathbb{C} / \mathbb{Z},
$$

and

$$
\operatorname{LFie}(W)=\bigoplus_{\alpha \in \mathbb{C} / \mathbb{Z}} \operatorname{LFie}_{\alpha}(W) .
$$

The elements of $\operatorname{LFie}(W)$ are called logarithmic (quantum) fields on $W$, and are denoted as $a(\zeta, z)$ or $a(z)$ for short.

By definition, every logarithmic field $a(z)$ is a finite sum of elements from the spaces $\operatorname{LFie}_{\alpha}(W)$. The composition of two logarithmic fields $a \in$ $\mathrm{LFie}_{\alpha}(W)$ and $b \in \mathrm{LFie}_{\beta}(W)$ is the linear map

$$
a\left(z_{1}\right) b\left(z_{2}\right): W \rightarrow\left(W\left[\zeta_{1}\right]\left[\left[z_{1}\right]\right] z_{1}^{-\alpha}\right)\left[\zeta_{2}\right]\left[\left[z_{2}\right]\right] z_{2}^{-\beta} .
$$

Definition 3.2. A pair of logarithmic fields $a, b$ is called local if

$$
z_{12}^{N} a\left(z_{1}\right) b\left(z_{2}\right)=z_{12}^{N} b\left(z_{2}\right) a\left(z_{1}\right), \quad z_{12}=z_{1}-z_{2},
$$

for some integer $N \geq 0$.

For every $v \in W$, the powers of $z_{2}$ in $a\left(z_{1}\right) b\left(z_{2}\right) v$ belong to the union of finitely many sets of the form $\gamma+\mathbb{Z}_{+}(\gamma \in \mathbb{C})$. If $a\left(z_{1}\right)$ and $b\left(z_{2}\right)$ are local, then

$$
z_{12}^{N} a\left(z_{1}\right) b\left(z_{2}\right) v=z_{12}^{N} b\left(z_{2}\right) a\left(z_{1}\right) v
$$

satisfies this property both for the powers of $z_{1}$ and $z_{2}$. In fact, when $a \in \operatorname{LFie}_{\alpha}(W)$ and $b \in \operatorname{LFie}_{\beta}(W)$ are local, both sides of this equation belong to the space

$$
W\left[\zeta_{1}, \zeta_{2}\right]\left[\left[z_{1}, z_{2}\right]\right] z_{1}^{-\alpha} z_{2}^{-\beta}
$$


3.2. $n$-th products. Now we define an operation on local logarithmic fields, which provides an algebraic formulation of the operator product expansion (cf. [BN, $\mathrm{BK2}, \mathrm{BM}]$ ).

Definition 3.3. For $n \in \mathbb{Z}$, the $n$-th product $a_{(n)} b$ of two local logarithmic fields $a, b$ is defined by:

$$
\left(a_{(n)} b\right)(\zeta, z) v=\left.D_{z_{1}}^{(N-1-n)}\left(z_{12}^{N} a\left(\zeta_{1}, z_{1}\right) b\left(\zeta_{2}, z_{2}\right) v\right)\right|_{\substack{z_{1}=z_{2}=z \\ \zeta_{1}=\zeta_{2}=\zeta}}
$$

for $v \in W$ and $n \leq N-1$. For $n \geq N$, let $a_{(n)} b=0$. As before, we will supress the dependence on $\zeta$ and understand that setting $z_{1}=z$ automatically sets $\zeta_{1}=\zeta$.

Note that $a_{(n)} b$ is again a logarithmic field, and it does not depend on the choice of $N$ satisfying (3.1). Moreover, $a_{(n)} b \in \mathrm{LFie}_{\alpha+\beta}(W)$ if $a \in \mathrm{LFie}_{\alpha}(W)$ and $b \in \operatorname{LFie}_{\beta}(W)$. Using the Leibniz rule, one can derive from (3.2) the following properties:

$$
\begin{aligned}
\left(D_{z} a\right)_{(n)} b & =-n a_{(n-1)} b, \\
D_{z}\left(a_{(n)} b\right) & =\left(D_{z} a\right)_{(n)} b+a_{(n)}\left(D_{z} b\right), \\
\partial_{\zeta}\left(a_{(n)} b\right) & =\left(\partial_{\zeta} a\right)_{(n)} b+a_{(n)}\left(\partial_{\zeta} b\right) .
\end{aligned}
$$

We also have an analog of Dong's Lemma (cf. [Li1, Li2, K2]).

Lemma 3.4. Let $a, b, c$ be logarithmic fields such that the pairs $(a, b),(a, c)$, $(b, c)$ are local. Then $a_{(n)} b$ and $c$ are local for all $n \in \mathbb{Z}$.

Proof. For some sufficiently large $N$, we have (3.1) and

$$
z_{13}^{N} a\left(z_{1}\right) c\left(z_{3}\right)=z_{13}^{N} c\left(z_{3}\right) a\left(z_{1}\right), \quad z_{23}^{N} b\left(z_{2}\right) c\left(z_{3}\right)=z_{23}^{N} c\left(z_{3}\right) b\left(z_{2}\right) .
$$

Using the Leibniz rule, we find for $n^{\prime}=N-1-n \geq 0$ and $v \in W$,

$$
\begin{gathered}
z_{23}^{2 N+n^{\prime}}\left(a_{(n)} b\right)\left(z_{2}\right) c\left(z_{3}\right) v=\left.\left(z_{13}^{N+n^{\prime}} D_{z_{1}}^{\left(n^{\prime}\right)}\left(z_{12}^{N} z_{23}^{N} a\left(z_{1}\right) b\left(z_{2}\right) c\left(z_{3}\right) v\right)\right)\right|_{z_{1}=z_{2}} \\
=\left.\sum_{i=0}^{n^{\prime}}(-1)^{n^{\prime}-i}\left(\begin{array}{c}
N+n^{\prime} \\
n^{\prime}-i
\end{array}\right) D_{z_{1}}^{(i)}\left(z_{13}^{N+i} z_{12}^{N} z_{23}^{N} a\left(z_{1}\right) b\left(z_{2}\right) c\left(z_{3}\right) v\right)\right|_{z_{1}=z_{2}} .
\end{gathered}
$$

We can move $c\left(z_{3}\right)$ to the left of $a\left(z_{1}\right) b\left(z_{2}\right)$ inside the parentheses, and then rewrite the whole expression back as $z_{23}^{2 N+n^{\prime}} c\left(z_{3}\right)\left(a_{(n)} b\right)\left(z_{2}\right) v$.

3.3. Normally ordered products and propagators. For $\alpha \in \mathbb{C} / \mathbb{Z}$, pick the unique representative $\alpha_{0} \in \alpha$ with $-1<\operatorname{Re} \alpha_{0} \leq 0$. Every logarithmic field $a \in \mathrm{LFie}_{\alpha}(W)$ can be expanded as

$$
a(\zeta, z)=\sum_{i \in \mathbb{Z}} a_{i}(\zeta) z^{-i-\alpha_{0}}, \quad a_{i}(\zeta) \in \operatorname{Hom}_{\mathbb{C}}(W, W[\zeta]),
$$


where for each $v \in W$ we have $a_{i}(\zeta) v=0$ for sufficiently large $i$. The annihilation and creation parts of $a(z)$ are defined respectively as

$$
\begin{aligned}
& a(z)_{-}=a(\zeta, z)_{-}=\sum_{i=1}^{\infty} a_{i}(\zeta) z^{-i-\alpha_{0}} \\
& a(z)_{+}=a(\zeta, z)_{+}=\sum_{i=-\infty}^{0} a_{i}(\zeta) z^{-i-\alpha_{0}} .
\end{aligned}
$$

These are extended by linearity to all $a \in \operatorname{LFie}(W)$. In other words, $a(z)_{-}$ is the part of $a(z)$ containing only $z^{\gamma}$ with $\operatorname{Re} \gamma<0$, while in $a(z)+$ we have only $z^{\gamma}$ with $\operatorname{Re} \gamma \geq 0$.

Definition 3.5. The normally ordered product of two logarithmic fields $a\left(z_{1}\right), b\left(z_{2}\right)$ is defined by:

$$
: a\left(z_{1}\right) b\left(z_{2}\right):=a\left(z_{1}\right)_{+} b\left(z_{2}\right)+b\left(z_{2}\right) a\left(z_{1}\right)_{-} .
$$

Their propagator is:

$$
P\left(a, b ; z_{1}, z_{2}\right)=\left[a\left(z_{1}\right)_{-}, b\left(z_{2}\right)\right]=a\left(z_{1}\right) b\left(z_{2}\right)-: a\left(z_{1}\right) b\left(z_{2}\right): .
$$

Just like for usual quantum fields (see, e.g., [K2]), it is easy to check that $: a\left(z_{1}\right) b\left(z_{2}\right)$ : is well defined for $z_{1}=z_{2}$ and $: a(z) b(z)$ : is again a logarithmic field.

Proposition 3.6. Let $a$ and $b$ be two local logarithmic fields, and $N$ be from (3.1). Then for $0 \leq n \leq N-1$ and $k \geq 0$, we have:

$$
\begin{aligned}
\left(a_{(n)} b\right)(z) & =\left.D_{z_{1}}^{(N-1-n)}\left(z_{12}^{N} P\left(a, b ; z_{1}, z_{2}\right)\right)\right|_{z_{1}=z_{2}=z}, \\
\left(a_{(-k-1)} b\right)(z) & =:\left(D_{z}^{(k)} a(z)\right) b(z):+\left.D_{z_{1}}^{(N+k)}\left(z_{12}^{N} P\left(a, b ; z_{1}, z_{2}\right)\right)\right|_{z_{1}=z_{2}=z} .
\end{aligned}
$$

Proof. The proof is a straightforward calculation using

$$
z_{12}^{N} a\left(z_{1}\right) b\left(z_{2}\right)=z_{12}^{N}: a\left(z_{1}\right) b\left(z_{2}\right):+z_{12}^{N} P\left(a, b ; z_{1}, z_{2}\right)
$$

and the fact that $: a\left(z_{1}\right) b\left(z_{2}\right)$ : is well defined for $z_{1}=z_{2}$.

3.4. Local collections of logarithmic fields. The identity operator $I$ is local with any other logarithmic field $a$, and satisfies

$$
a_{(n)} I=0, \quad a_{(-n-1)} I=D_{z}^{(n)} a, \quad n \geq 0 .
$$

Let $\mathcal{W} \subset \operatorname{LFie}(W)$ be a local collection, i.e., such that every pair $a, b \in \mathcal{W}$ is local. We can add $I$ to $\mathcal{W}$ and still have a local collection. If a pair $(a, b)$ is local, then $\left(D_{\zeta} a, b\right)$ is also local; thus the $\mathbb{C}\left[D_{\zeta}\right]$-module generated by $\mathcal{W}$ is again local. Due to Lemma 3.4, the smallest subspace $\overline{\mathcal{W}} \subset \operatorname{LFie}(W)$ containing $\mathcal{W} \cup\{I\}$ and closed under $D_{\zeta}$ and all $n$-th products is a local collection. Similarly to [Li1, Li2], we have the following result.

Theorem 3.7. The $n$-th products endow the space $\overline{\mathcal{W}}$ with the structure of a vertex algebra with a vacuum vector $I$ and translation operator $D_{z}$. 
Proof. The state-field correspondence $Y: \overline{\mathcal{W}} \rightarrow \operatorname{Fie}(\overline{\mathcal{W}})$ is given by

$$
Y(a, x) b=\sum_{n \in \mathbb{Z}} x^{-n-1}\left(a_{(n)} b\right), \quad a, b \in \overline{\mathcal{W}}
$$

where the formal variable is now denoted by $x$, and $a_{(n)} b$ is again defined by (3.2). Due to the already established properties of the $n$-th products, it only remains to prove that $Y(a, x)$ and $Y(b, x)$ are local for $a, b \in \overline{\mathcal{W}}$.

When we need to specify the formal variable in the fields $a$ and $b$, we will write $Y$ as

$$
Y(a(z), x) b(z)=\sum_{n \in \mathbb{Z}} x^{-n-1}\left(a_{(n)} b\right)(z) .
$$

It follows immediately from (3.2) that for all $v \in W$,

$$
(Y(a(z), x) b(z)) v=\left.x^{-N} e^{x D_{z_{1}}}\left(z_{12}^{N} a\left(z_{1}\right) b\left(z_{2}\right) v\right)\right|_{z_{1}=z_{2}=z},
$$

where, as before, $N$ is such that the locality (3.1) holds. For brevity, through the rest of the proof we will omit the vector $v$.

Consider $a, b, c \in \overline{\mathcal{W}}$, and take $N$ to be an even number such that (3.1) holds for the pairs $(a, b),(a, c)$ and $(b, c)$. By the proof of Lemma 3.4 for any $k \leq N-1$, the pair $\left(a, b_{(k)} c\right)$ satisfies (3.1) with $N$ replaced by $2 N+k^{\prime}$ where $k^{\prime}=N-1-k \geq 0$. Therefore,

$$
\begin{aligned}
& Y\left(a(z), x_{1}\right)\left(b_{(k)} c\right)(z) \\
& \quad=\left.x_{1}^{-2 N-k^{\prime}} e^{x_{1} D_{z_{1}}}\left(z_{13}^{2 N+k^{\prime}} D_{z_{2}}^{\left(k^{\prime}\right)}\left(z_{23}^{N} a\left(z_{1}\right) b\left(z_{2}\right) c\left(z_{3}\right)\right)\right)\right|_{z_{1}=z_{2}=z_{3}=z} .
\end{aligned}
$$

Summing over $k$, we obtain:

$$
\begin{aligned}
& x_{1}^{2 N} x_{2}^{N} Y\left(a(z), x_{1}\right) Y\left(b(z), x_{2}\right) c(z) \\
& =\left.\sum_{k^{\prime}=0}^{\infty}\left(\frac{x_{2}}{x_{1}}\right)^{k^{\prime}} e^{x_{1} D_{z_{1}}}\left(z_{13}^{2 N+k^{\prime}} D_{z_{2}}^{\left(k^{\prime}\right)}\left(z_{23}^{N} a\left(z_{1}\right) b\left(z_{2}\right) c\left(z_{3}\right)\right)\right)\right|_{z_{1}=z_{2}=z_{3}=z} .
\end{aligned}
$$

Notice that here $z_{13}^{2 N+k^{\prime}}$ can be replaced by $z_{13}^{N} z_{12}^{N+k^{\prime}}$. Consider the linear operator

$$
A_{2}=\sum_{k^{\prime}=0}^{\infty}\left(\frac{x_{2}}{x_{1}}\right)^{k^{\prime}} z_{12}^{k^{\prime}} D_{z_{2}}^{\left(k^{\prime}\right)}=\left(1-\frac{x_{2}}{x_{1}}\right)^{z_{21} D_{z_{2}}},
$$

where we applied the well-known identity $z^{k} \partial_{z}^{(k)}=\left(\begin{array}{c}z \partial_{z} \\ k\end{array}\right)$.

Then

and we have

$$
z_{12}^{N} A_{2}=\left(1-\frac{x_{2}}{x_{1}}\right)^{-N} A_{2} \circ z_{12}^{N}
$$

$$
\begin{aligned}
x_{1}^{2 N} & x_{2}^{N} Y\left(a(z), x_{1}\right) Y\left(b(z), x_{2}\right) c(z) \\
& =\left.e^{x_{1} D_{z_{1}}}\left(z_{13}^{N} z_{12}^{N} A_{2}\left(z_{23}^{N} a\left(z_{1}\right) b\left(z_{2}\right) c\left(z_{3}\right)\right)\right)\right|_{z_{1}=z_{2}=z_{3}=z} \\
& =\left.\left(1-\frac{x_{2}}{x_{1}}\right)^{-N} e^{x_{1} D_{z_{1}}} A_{2}\left(z_{13}^{N} z_{12}^{N} z_{23}^{N} a\left(z_{1}\right) b\left(z_{2}\right) c\left(z_{3}\right)\right)\right|_{z_{1}=z_{2}=z_{3}=z} .
\end{aligned}
$$


Now observe that

$$
e^{x_{1} D_{z_{1}}} A_{2}=\sum_{k=0}^{\infty}\left(\frac{x_{2}}{x_{1}}\right)^{k}\left(x_{1}+z_{12}\right)^{k} D_{z_{2}}^{(k)} e^{x_{1} D_{z_{1}}}
$$

becomes $e^{x_{2} D_{z_{2}}} e^{x_{1} D_{z_{1}}}$ after setting $z_{1}=z_{2}$. Therefore,

$$
\begin{aligned}
& x_{1}^{N} x_{2}^{N}\left(x_{1}-x_{2}\right)^{N} Y\left(a(z), x_{1}\right) Y\left(b(z), x_{2}\right) c(z) \\
& =\left.e^{x_{1} D_{z_{1}}+x_{2} D_{z_{2}}}\left(z_{13}^{N} z_{12}^{N} z_{23}^{N} a\left(z_{1}\right) b\left(z_{2}\right) c\left(z_{3}\right)\right)\right|_{z_{1}=z_{2}=z_{3}=z} .
\end{aligned}
$$

This implies the locality of $Y(a, x)$ and $Y(b, x)$, thus completing the proof of the theorem.

It follows from (3.2) and $\left[D_{\zeta}, D_{z}\right]=-D_{z}$ that

$$
D_{\zeta}\left(a_{(n)} b\right)=\left(D_{\zeta} a\right)_{(n)} b+a_{(n)}\left(D_{\zeta} b\right)+(n+1)\left(a_{(n)} b\right) .
$$

Hence, $e^{2 \pi \mathrm{i} D_{\zeta}}$ is an automorphism of the vertex algebra $\overline{\mathcal{W}}$. It acts exactly as the monodromy operator around 0 , sending $\zeta$ to $\zeta+2 \pi \mathrm{i}$ and $z^{\gamma}$ to $e^{2 \pi \mathrm{i} \gamma} z^{\gamma}$. Note that $e^{2 \pi \mathrm{i} D_{\zeta}}=e^{2 \pi \mathrm{i} z \partial_{z}} e^{2 \pi \mathrm{i} \partial_{\zeta}}$ and $e^{2 \pi \mathrm{i} z \partial_{z}} \in \operatorname{Aut}(\overline{\mathcal{W}}), \partial_{\zeta} \in \operatorname{Der}(\overline{\mathcal{W}})$.

\section{Definition of twisted modules}

From now on, $V$ will be a vertex algebra and $\varphi$ an automorphism of $V$, which is not necessarily of finite order. In this section, we introduce the notion of a $\varphi$-twisted $V$-module and establish some of its basic properties. We continue to use the notation from Section 3 .

4.1. $\varphi$-twisted modules. The following is the main object of the paper.

Definition 4.1. A $\varphi$-twisted $V$-module is a vector space $W$, equipped with a linear map $Y: V \rightarrow \operatorname{LFie}(W)$ such that $Y(\mathbf{1})=I$ is the identity operator, $Y(V)$ is a local collection,

$$
Y(\varphi a, z)=e^{2 \pi \mathrm{i} D_{\zeta}} Y(a, z),
$$

and

$$
Y\left(a_{(n)} b, z\right)=Y(a, z)_{(n)} Y(b, z)
$$

for all $a, b \in V, n \in \mathbb{Z}$. We will call (4.1) the $\varphi$-equivariance, and (4.2) the $n$-th product identity.

Remark 4.2. Y.-Z. Huang has introduced in [H] a notion of a $\varphi$-twisted $V$ module $W$, which is more restrictive than ours (in particular, it assumes certain gradings of $V$ and $W$ ). One can show that every $\varphi$-twisted module in the sense of $[\mathrm{H}]$ satisfies our definition. Conversely, as will be indicated below, some assumptions of [H, Definition 3.1] also hold in our case.

Remark 4.3. S.-Q. Liu, D. Yang, and Y. Zhang have introduced in [LYZ] a notion of a $\varphi$-twisted $V$-module, which has some similarities to ours but also important differences. In particular, it involves vectors in $V \otimes \mathbb{C}^{d}$ and a certain $d \times d$ matrix associated to a Frobenius manifold of dimension $d$. 
As a consequence of (4.2) and $T a=a_{(-2)} \mathbf{1}$, we have (cf. [] $)$ :

$$
Y(T a, z)=D_{z} Y(a, z), \quad a \in V .
$$

Eqs. (4.1), (4.2) can be stated equivalently that $Y: V \rightarrow \overline{\mathcal{W}}$ is a vertex algebra homomorphism compatible with the automorphisms $\varphi$ and $e^{2 \pi \mathrm{i} D_{\zeta}}$, where $\overline{\mathcal{W}}=Y(V)$ (see Theorem 3.7).

Example 4.4. Let $W$ be a vector space, $\mathcal{W} \subset \operatorname{LFie}(W)$ a local collection, $\overline{\mathcal{W}}$ be the vertex algebra generated by $\mathcal{W}$, and $\varphi=e^{2 \pi i D_{\zeta}} \in \operatorname{Aut}(\overline{\mathcal{W}})$ (see Theorem 3.7). Then the identity map $Y: \overline{\mathcal{W}} \rightarrow \operatorname{LFie}(W), Y(a, z)=a(z)$, provides $W$ with the structure of a $\varphi$-twisted $\overline{\mathcal{W}}$-module (cf. [Li1, Li2]).

Remark 4.5. The space $V^{\varphi}$ of $\varphi$-invariants (i.e., $a \in V$ such that $\varphi a=a$ ) is a subalgebra of $V$. The restriction of any $\varphi$-twisted $V$-module to $V^{\varphi}$ is a (untwisted) $V^{\varphi}$-module.

4.2. Locally finite automorphisms. A linear operator $\varphi$ on $V$ is called $l o$ cally finite if every $a \in V$ is contained in some finite-dimensional $\varphi$-invariant subspace of $V$ (see [K1, Chapter 3]). In particular, this holds when $V$ is a direct sum of finite-dimensional $\varphi$-invariant subspaces, as is assumed in $[\mathrm{H}]$. A linear operator $\mathcal{N}$ on $V$ is called locally nilpotent if for every $a \in V$ we have $\mathcal{N}^{l} a=0$ for some $l \geq 1$. The next lemma is standard.

Lemma 4.6. Every invertible locally finite linear operator $\varphi$ can be written uniquely in the form $\varphi=\sigma e^{-2 \pi \mathrm{i} \mathcal{N}}$, where $\sigma$ is semisimple, $\mathcal{N}$ is locally nilpotent and $\sigma \mathcal{N}=\mathcal{N} \sigma$. Furthermore, if $\varphi \in \operatorname{Aut}(V)$, then $\sigma \in \operatorname{Aut}(V)$ and $\mathcal{N} \in \operatorname{Der}(V)$.

Proof. Fix $a \in V$ and a finite-dimensional subspace $U \subset V$ such that $a \in U$ and $\varphi(U) \subset U$. Then the restriction $\left.\varphi\right|_{U}$ has the desired decomposition (Jordan-Chevalley decomposition). If we have another such subspace $U^{\prime} \supset$ $U$, it will give rise to the same $\sigma$ and $\mathcal{N}$ when restricted to $U$. Therefore, $\sigma$ and $\mathcal{N}$ are uniquely defined and are independent of the choice of $U$.

Let $\varphi \in \operatorname{Aut}(V)$, and $a, b \in V$ be such that $(\varphi-\lambda)^{l} a=(\varphi-\mu)^{l} b=0$ for some $\lambda, \mu \in \mathbb{C}$ and $l \geq 1$. Then $\sigma a=\lambda a$ and $\sigma b=\mu b$. The identity

$$
\varphi \otimes \varphi-\lambda \otimes \mu=(\varphi-\lambda) \otimes \varphi+\lambda \otimes(\varphi-\mu)
$$

then implies that

$$
(\varphi-\lambda \mu)^{m}\left(a_{(n)} b\right)=\sum_{k=0}^{m}\left(\begin{array}{c}
m \\
k
\end{array}\right)\left((\varphi-\lambda)^{k} \lambda^{m-k} a\right)_{(n)}\left(\varphi^{k}(\varphi-\mu)^{m-k} b\right)=0
$$

for $m \geq 2 l-1$. Therefore, $\sigma\left(a_{(n)} b\right)=\lambda \mu\left(a_{(n)} b\right)$ and $\sigma \in \operatorname{Aut}(V)$.

To prove that $\mathcal{N} \in \operatorname{Der}(V)$, consider the expression

$$
e^{2 \pi \mathrm{i} x \mathcal{N}}\left(a_{(n)} b\right)-\left(e^{2 \pi \mathrm{i} x \mathcal{N}} a\right)_{(n)}\left(e^{2 \pi \mathrm{i} x \mathcal{N}} b\right),
$$

which is a polynomial in $x$. This polynomial vanishes at all $k \in \mathbb{Z}$, since $\left(\varphi \sigma^{-1}\right)^{k} \in \operatorname{Aut}(V)$. Taking $\partial_{x}$ at $x=0$, we obtain that $\mathcal{N} \in \operatorname{Der}(V)$. 
We will say that $\varphi$ is locally finite on $a \in V$ if there is a finite-dimensional subspace $U \subset V$ such that $a \in U$ and $\varphi(U) \subset U$.

Lemma 4.7. The set $\bar{V}$ of all $a \in V$, on which $\varphi$ is locally finite, is the maximal $\varphi$-invariant subspace $\bar{V} \subset V$ such that the restriction $\left.\varphi\right|_{\bar{V}}$ is locally finite. Moreover, $\bar{V}$ is a subalgebra of $V$.

Proof. This follows easily from the definitions. Indeed, let $U$ and $U^{\prime}$ be finite-dimensional $\varphi$-invariant subspaces such that $a \in U, b \in U^{\prime}$. Then $a+\lambda b \in U+U^{\prime}$ and $a_{(n)} b \in U_{(n)} U^{\prime}$ for all $\lambda \in \mathbb{C}, n \in \mathbb{Z}$.

4.3. Consequences of local finiteness. Consider again an automorphism $\varphi$ of $V$ and a $\varphi$-twisted $V$-module $W$. Let $\bar{V} \subset V$ be the maximal subalgebra on which $\varphi$ is locally finite (see Lemma 4.7). Write $\left.\varphi\right|_{\bar{V}}=\sigma e^{-2 \pi \mathrm{i} \mathcal{N}}$, as in Lemma 4.6, where $\sigma \in \operatorname{Aut}(\bar{V})$ is semisimple and $\mathcal{N} \in \operatorname{Der}(\bar{V})$ is locally nilpotent.

Lemma 4.8. For all $a \in \bar{V}$, we have

$$
Y(\sigma a, z)=e^{2 \pi \mathrm{i} z \partial_{z}} Y(a, z), \quad Y(\mathcal{N} a, z)=-\partial_{\zeta} Y(a, z) .
$$

Proof. This follows from the uniqueness of $\sigma$ and $\mathcal{N}$ from Lemma 4.6, since the semisimple part of $e^{2 \pi \mathrm{i} D_{\zeta}}$ is $e^{2 \pi \mathrm{i} z \partial_{z}}$ and the corresponding locally nilpotent operator is $-\partial_{\zeta}$.

In particular, since $\mathcal{N}$ is locally nilpotent, we see from (4.4) that each logarithmic field $Y(a, z)$ is a polynomial in $\zeta$ for $a \in \bar{V}$ (cf. [H]). Introduce the linear map $z^{\mathcal{N}}$ from $\bar{V}$ to $\bar{V}[\zeta]$, given by

$$
z^{\mathcal{N}} a=e^{\zeta \mathcal{N}} a \in \bar{V}[\zeta], \quad a \in \bar{V},
$$

and let

$$
X(a, z)=Y\left(z^{\mathcal{N}} a, z\right), \quad Y(a, z)=X\left(z^{-\mathcal{N}} a, z\right), \quad a \in \bar{V} .
$$

Note that, since $\mathcal{N} \in \operatorname{Der}(\bar{V})$, we have

$$
z^{\mathcal{N}}\left(a_{(n)} b\right)=\left(z^{\mathcal{N}} a\right)_{(n)}\left(z^{\mathcal{N}} b\right), \quad a, b \in \bar{V}, \quad n \in \mathbb{Z} .
$$

Lemma 4.9. With the above notation, we have $X(a, z)=\left.Y(a, z)\right|_{\zeta=0}$ for all $a \in \bar{V}$. Furthermore, $X(\bar{V})$ is a local collection of fields.

Proof. Using (4.4), we find

$$
\begin{aligned}
\partial_{\zeta} X(a, z) & =Y\left(\mathcal{N} z^{\mathcal{N}} a, z\right)+\left.\partial_{\zeta} Y\left(a^{\prime}, z\right)\right|_{a^{\prime}=z^{\mathcal{N}} a} \\
& =Y\left(\mathcal{N} z^{\mathcal{N}} a, z\right)-\left.Y\left(\mathcal{N} a^{\prime}, z\right)\right|_{a^{\prime}=z^{\mathcal{N}} a}=0 .
\end{aligned}
$$

Then $X(a, z)=\left.X(a, z)\right|_{\zeta=0}=\left.Y(a, z)\right|_{\zeta=0}$. Setting $\zeta_{1}=\zeta_{2}=0$ in (3.1), we see that all the fields $X(a, z)$ are local.

Remark 4.10. The kernel $V^{\mathcal{N}} \subset \bar{V}$ of $\mathcal{N}$ is a subalgebra of $V$. The restriction of any $\varphi$-twisted $V$-module to $V^{\mathcal{N}}$ is a $\sigma$-twisted $V^{\mathcal{N}}$-module. 
4.4. $\mathcal{D}$-twisted modules. When $\mathcal{N}$ is not locally nilpotent, we might not be able to exponentiate it. However, (4.4) still makes sense, suggesting the following more general notion, which we plan to investigate in the future.

Definition 4.11. Let $V$ be a vertex algebra and $\mathcal{D} \in \operatorname{Der}(V)$. A $\mathcal{D}$-twisted $V$-module is a vector space $W$, equipped with a linear map

$$
Y: V \rightarrow \operatorname{Hom}_{\mathbb{C}}(W, W[[\zeta]]((z)))
$$

such that

$$
Y(\mathcal{D} a, z)=-\partial_{\zeta} Y(a, z), \quad a \in V,
$$

$Y(\mathbf{1})=I$ is the identity operator, $Y(V)$ is a local collection, and the $n$-th product identity (4.2) holds.

Observe that, in comparison to Definition 4.1, here we allow the logarithmic fields $Y(a, z)$ to have coefficients formal power series in $\zeta$. However, all results of Section 3 still hold in this case. When $\mathcal{D}$ is locally finite, then $\varphi=e^{-2 \pi \mathrm{i} \mathcal{D}} \in \operatorname{Aut}(V)$ is locally finite and the notion of a $\mathcal{D}$-twisted module is equivalent to that of a $\varphi$-twisted module.

Remark 4.12. For every $\mathcal{D} \in \operatorname{Der}(V)$, the kernel $V^{\mathcal{D}}$ of $\mathcal{D}$ is a subalgebra of $V$. The restriction of any $\mathcal{D}$-twisted $V$-module to $V^{\mathcal{D}}$ is a (untwisted) $V^{\mathcal{D}}$-module.

\section{BORCHERDS IDENTITY FOR TWISTED MODUlES}

In this section, we derive a Borcherds identity for twisted modules and, as a consequence, a commutator formula. We prove that the Borcherds identity can replace the locality and $n$-th product identity in the definition of a twisted module.

5.1. Modes in a twisted module. Throughout this section, $V$ will be a vertex algebra, $\varphi$ a locally finite automorphism of $V$, and $W$ a $\varphi$-twisted $V$ module. We will again write $\varphi=\sigma e^{-2 \pi \mathrm{i} \mathcal{N}}$ with commuting semisimple $\sigma \in$ $\operatorname{Aut}(V)$ and locally nilpotent $\mathcal{N} \in \operatorname{Der}(V)$ (see Section 4.3). In particular, $\bar{V}=V$ as $\varphi$ is locally finite.

We will denote by

$$
V_{\alpha}=\left\{a \in V \mid \sigma a=e^{-2 \pi \mathrm{i} \alpha} a\right\}, \quad \alpha \in \mathbb{C} / \mathbb{Z},
$$

the eigenspaces of $\sigma$. Then by (4.4) we have $Y\left(V_{\alpha}\right) \subset \operatorname{LFie}_{\alpha}(W)$, which means that all powers of $z$ in $Y(a, z)$ belong to the coset $-\alpha$ for $a \in V_{\alpha}$.

Definition 5.1. For $a \in V_{\alpha}, \alpha \in \mathbb{C} / \mathbb{Z}$ and $m \in \alpha$, the $(m+\mathcal{N})$-th mode of $a$ is defined as

$$
a_{(m+\mathcal{N})}=\operatorname{Res}_{z} z^{m} X(a, z)=\operatorname{Res}_{z} Y\left(z^{m+\mathcal{N}} a, z\right) \in \operatorname{End}(W),
$$

where $z^{m+\mathcal{N}}=z^{m} e^{\zeta \mathcal{N}}$. 
Since $\mathcal{N}\left(V_{\alpha}\right) \subset V_{\alpha}$ and $X(a, z)$ is independent of $\zeta$, we have

$$
X(a, z)=\sum_{m \in \alpha} a_{(m+\mathcal{N})} z^{-m-1}, \quad a \in V_{\alpha} .
$$

We can recover the field $Y(a, z)$ from the modes of $\mathcal{N}^{l} a(l \geq 0)$ as follows:

$$
\begin{aligned}
Y(a, z) & =X\left(e^{-\zeta \mathcal{N}} a, z\right)=\sum_{m \in \alpha}\left(e^{-\zeta \mathcal{N}} a\right)_{(m+\mathcal{N})} z^{-m-1} \\
& =\sum_{m \in \alpha}\left(z^{-m-1-\mathcal{N}} a\right)_{(m+\mathcal{N})}, \quad a \in V_{\alpha} .
\end{aligned}
$$

Note that for every $a \in V_{\alpha}, m \in \alpha$ and $v \in W$, there is an integer $L$ such that

$$
a_{(m+i+\mathcal{N})} v=0 \text { for all } i \in \mathbb{Z}, i \geq L .
$$

5.2. Borcherds identity. Now we can derive the main identity satisfied by the modes.

Theorem 5.2. Let $V$ be a vertex algebra, $\varphi$ a locally finite automorphism of $V$, and $W$ a $\varphi$-twisted $V$-module. Then we have the Borcherds identity

$$
\begin{gathered}
\sum_{i=0}^{\infty}(-1)^{i}\left(\begin{array}{c}
n \\
i
\end{array}\right) a_{(m+n-i+\mathcal{N})}\left(b_{(k+i+\mathcal{N})} v\right) \\
-\sum_{i=0}^{\infty}(-1)^{n+i}\left(\begin{array}{c}
n \\
i
\end{array}\right) b_{(k+n-i+\mathcal{N})}\left(a_{(m+i+\mathcal{N})} v\right) \\
\left.=\sum_{j=0}^{\infty}\left(\left(\begin{array}{c}
m+\mathcal{N} \\
j
\end{array}\right) a\right)_{(n+j)} b\right)_{(m+k-j+\mathcal{N})} v,
\end{gathered}
$$

for $a \in V_{\alpha}, b \in V_{\beta}, v \in W$, and $m \in \alpha, k \in \beta, n \in \mathbb{Z}$.

Proof. Notice that all sums in (5.3) are finite. Let $N$ be such that $\left(\mathcal{N}^{l} a\right)_{(j)} b=$ 0 for all $l \geq 0, j \geq N$. Then

$$
z_{12}^{N} Y\left(a, z_{1}\right) Y\left(b, z_{2}\right) v=z_{12}^{N} Y\left(b, z_{2}\right) Y\left(a, z_{1}\right) v,
$$

and let us denote both sides by $F\left(a, b ; z_{1}, z_{2}\right)$. Using the expansions (2.6) and (2.7), we compute for $n \leq N-1$ :

$$
\begin{gathered}
\iota_{z_{1}, z_{2}} z_{12}^{n} Y\left(a, z_{1}\right) Y\left(b, z_{2}\right) v-\iota_{z_{2}, z_{1}} z_{12}^{n} Y\left(b, z_{2}\right) Y\left(a, z_{1}\right) v \\
=F\left(a, b ; z_{1}, z_{2}\right)\left(\iota_{z_{1}, z_{2}}-\iota_{z_{2}, z_{1}}\right) z_{12}^{n-N} \\
=F\left(a, b ; z_{1}, z_{2}\right) \partial_{z_{2}}^{(N-1-n)} \delta\left(z_{1}, z_{2}\right) .
\end{gathered}
$$

Let us now replace in this equation $a$ with $z_{1}^{m+\mathcal{N}} a$ and $b$ with $z_{2}^{k+\mathcal{N}} b$ to get

$$
\begin{gathered}
\iota_{z_{1}, z_{2}} z_{12}^{n} z_{1}^{m} z_{2}^{k} X\left(a, z_{1}\right) X\left(b, z_{2}\right) v-\iota_{z_{2}, z_{1}} z_{12}^{n} z_{1}^{m} z_{2}^{k} X\left(b, z_{2}\right) X\left(a, z_{1}\right) v \\
=F\left(z_{1}^{m+\mathcal{N}} a, z_{2}^{k+\mathcal{N}} b ; z_{1}, z_{2}\right) \partial_{z_{2}}^{(N-1-n)} \delta\left(z_{1}, z_{2}\right) .
\end{gathered}
$$


If we then take $\operatorname{Res}_{z_{1}} \operatorname{Res}_{z_{2}}$ of the left-hand side of (5.6), we will obtain the left-hand side of (5.3), for any $n \in \mathbb{Z}$. By the property (2.8) of the delta function, if we take $\operatorname{Res}_{z_{1}}$ of the right-hand side of (5.6), we will get

$$
\left.\partial_{z_{1}}^{(N-1-n)} F\left(z_{1}^{m+\mathcal{N}} a, z_{2}^{k+\mathcal{N}} b ; z_{1}, z_{2}\right)\right|_{z_{1}=z_{2}} .
$$

In this formula, we can replace $\partial_{z_{1}}$ with $D_{z_{1}}$, because $F\left(z_{1}^{m+\mathcal{N}} a, z_{2}^{k+\mathcal{N}} b\right.$; $\left.z_{1}, z_{2}\right)$ is independent of $\zeta_{1}$. Then using the Leibniz rule, (3.2), (4.2) and (4.5), we obtain:

$$
\begin{aligned}
\sum_{j=0}^{N-1-n} & \left.D_{z_{1}}^{(N-1-n-j)} F\left(\left(\begin{array}{c}
m+\mathcal{N} \\
j
\end{array}\right) z_{2}^{m-j+\mathcal{N}} a, z_{2}^{k+\mathcal{N}} b ; z_{1}, z_{2}\right)\right|_{z_{1}=z_{2}} \\
& =\sum_{j=0}^{N-1-n} Y\left(\left(\left(\begin{array}{c}
m+\mathcal{N} \\
j
\end{array}\right) z_{2}^{m-j+\mathcal{N}} a\right)_{(n+j)}\left(z_{2}^{k+\mathcal{N}} b\right), z_{2}\right) v \\
& =\sum_{j=0}^{N-1-n} Y\left(z_{2}^{m+k-j+\mathcal{N}}\left(\left(\left(\begin{array}{c}
m+\mathcal{N} \\
j
\end{array}\right) a\right)_{(n+j)} b\right), z_{2}\right) v
\end{aligned}
$$

Now taking $\operatorname{Res}_{z_{2}}$ gives exactly the right-hand side of (5.3). This proves (5.3) in the case $n \leq N-1$. When $n \geq N$, the left-hand side of (5.5) is 0 . The right-hand side of (5.3) is also obviously 0 for $n \geq N$.

For $\alpha \in \mathbb{C} / \mathbb{Z}$, introduce the shifted delta function (cf. [BK1]):

$$
\begin{aligned}
\delta_{\alpha+\mathcal{N}}\left(z_{1}, z_{2}\right) & =\sum_{m \in \alpha} z_{1}^{-m-1-\mathcal{N}} z_{2}^{m+\mathcal{N}} \\
& =z_{1}^{-m} z_{2}^{m} \delta\left(z_{1}, z_{2}\right) e^{\left(\zeta_{2}-\zeta_{1}\right) \mathcal{N}}, \quad m \in \alpha,
\end{aligned}
$$

where $e^{\left(\zeta_{2}-\zeta_{1}\right) \mathcal{N}}$ is a linear map from $V$ to $V\left[\zeta_{1}, \zeta_{2}\right]$.

Proposition 5.3. The Borcherds identity (5.3) is equivalent to the equation

$$
\begin{gathered}
\iota_{z_{1}, z_{2}} z_{12}^{n} Y\left(a, z_{1}\right) Y\left(b, z_{2}\right) v-\iota_{z_{2}, z_{1}} z_{12}^{n} Y\left(b, z_{2}\right) Y\left(a, z_{1}\right) v \\
=\sum_{j=0}^{\infty} Y\left(\left(D_{z_{2}}^{(j)} \delta_{\alpha+\mathcal{N}}\left(z_{1}, z_{2}\right) a\right)_{(n+j)} b, z_{2}\right) v
\end{gathered}
$$

for $a \in V_{\alpha}, b \in V, v \in W$ and $n \in \mathbb{Z}$.

Proof. In the proof of Theorem 5.2 we saw that we can get the left-hand side of (5.3) from the left-hand side of (5.8) if we replace $a$ with $z_{1}^{m+\mathcal{N}} a, b$ with $z_{2}^{k+\mathcal{N}_{b}}$ and then take $\operatorname{Res}_{z_{1}} \operatorname{Res}_{z_{2}}$. Conversely, we can go back by summing over all $m, k$. Similarly, the right-hand side of (5.8), which is equal to

$$
\sum_{j=0}^{\infty} \sum_{m \in \alpha} Y\left(\left(\left(\begin{array}{c}
m+\mathcal{N} \\
j
\end{array}\right) z_{1}^{-m-1-\mathcal{N}} z_{2}^{m-j+\mathcal{N}} a\right)_{(n+j)} b, z_{2}\right) v,
$$

corresponds to the right-hand side of (5.3) . 
Remark 5.4. The Borcherds identity (5.8) remains true without the assumption that $\varphi$ is locally finite. However, it requires that $a \in V_{\alpha} \subset \bar{V}$, so $\varphi$ is locally finite on $a$.

Next, we show that the Borcherds identity can replace the locality and $n$-th product identity in the definition of a $\varphi$-twisted module.

Proposition 5.5. Let $V$ be a vertex algebra, $\varphi$ a locally finite automorphism, $W$ a vector space, and $Y: V \rightarrow \operatorname{LFie}(W)$ a linear map satisfying the $\varphi$-equivariance (4.1) and the Borcherds identity (5.8). Then $W$ is a $\varphi$-twisted $V$-module.

Proof. The proof follows by reversing the proofs of Theorem 5.2 and Proposition 5.3. Fix $a, b \in V$, and let $N \geq 0$ be such that $\left(\mathcal{N}^{l} a\right)_{(j)} b=0$ for all $l \geq 0, j \geq N$. Then setting $n=N$ in (5.8), we obtain the locality (5.4) for all $v \in W$.

Note that (4.2) is trivial for $n \geq N$. Suppose $n \leq N-1$ and denote both sides of (5.4) again by $F\left(a, b ; z_{1}, z_{2}\right)$. By (5.5) and (5.8),

$F\left(a, b ; z_{1}, z_{2}\right) \partial_{z_{2}}^{(N-1-n)} \delta\left(z_{1}, z_{2}\right)=\sum_{j=0}^{N-1-n} Y\left(\left(D_{z_{2}}^{(j)} \delta_{\alpha+\mathcal{N}}\left(z_{1}, z_{2}\right) a\right)_{(n+j)} b, z_{2}\right) v$.

Now if we replace $a$ with $z_{1}^{m+\mathcal{N}} a$, where $a \in V_{\alpha}, m \in \alpha$, we will have only integral powers of $z_{1}$ and no dependence on $\zeta_{1}$. Then take $\operatorname{Res}_{z_{1}}$ to obtain $\left.\partial_{z_{1}}^{(N-1-n)} F\left(z_{1}^{m+\mathcal{N}} a, b ; z_{1}, z_{2}\right)\right|_{z_{1}=z_{2}}=\sum_{j=0}^{N-1-n} Y\left(\left(D_{z_{2}}^{(j)}\left(z_{2}^{m+\mathcal{N}}\right) a\right)_{(n+j)} b, z_{2}\right) v$.

By the Leibniz rule, the left-hand side is equal to

$$
\left.\sum_{j=0}^{N-1-n} D_{z_{1}}^{(N-1-n-j)} F\left(D_{z_{2}}^{(j)}\left(z_{2}^{m+\mathcal{N}}\right) a, b ; z_{1}, z_{2}\right)\right|_{z_{1}=z_{2}} .
$$

Then by induction on $N-1-n$, it follows that

$$
\left.D_{z_{1}}^{(N-1-n)} F\left(a, b ; z_{1}, z_{2}\right)\right|_{z_{1}=z_{2}}=Y\left(a_{(n)} b, z_{2}\right),
$$

which is exactly (4.2).

5.3. Commutator formulas. Setting $n=0$ in the Borcherds identity (5.3), we obtain the commutator formula

$$
\left[a_{(m+\mathcal{N})}, b_{(k+\mathcal{N})}\right]=\sum_{j=0}^{\infty}\left(\left(\left(\begin{array}{c}
m+\mathcal{N} \\
j
\end{array}\right) a\right)_{(j)} b\right)_{(m+k-j+\mathcal{N})},
$$

where $a \in V_{\alpha}, b \in V_{\beta}, m \in \alpha, k \in \beta$. Similarly, from (5.8) we have:

$$
\left[Y\left(a, z_{1}\right), Y\left(b, z_{2}\right)\right]=\sum_{j=0}^{\infty} Y\left(\left(D_{z_{2}}^{(j)} \delta_{\alpha+\mathcal{N}}\left(z_{1}, z_{2}\right) a\right)_{(j)} b, z_{2}\right)
$$


for all $a \in V_{\alpha}$ and $b \in V$. Extracting the coefficient of $z_{1}^{-m-1-\mathcal{N}} a$, we deduce another useful formula:

$$
\left[a_{(m+\mathcal{N})}, Y(b, z)\right]=\sum_{j=0}^{\infty} Y\left(\left(\left(\begin{array}{c}
m+\mathcal{N} \\
j
\end{array}\right) z^{m-j+\mathcal{N}} a\right)_{(j)} b, z\right) .
$$

As in Remark 5.4, equations (5.10) and (5.11) hold without the assumption that $\varphi$ is locally finite, but they require $a \in V_{\alpha} \subset \bar{V}$.

From (5.10) we can derive a formula for the propagator $P\left(a, b ; z_{1}, z_{2}\right)$ of $Y\left(a, z_{1}\right)$ and $Y\left(b, z_{2}\right)$ (see Section 3.4). Recall that $\alpha_{0} \in \alpha$ is such that $-1<\operatorname{Re} \alpha_{0} \leq 0$.

Lemma 5.6. For any $a \in V_{\alpha}$ and $b \in V$, we have

$$
z_{12}^{N} P\left(a, b ; z_{1}, z_{2}\right)=\sum_{j=0}^{N-1} \sum_{i=0}^{j} z_{12}^{N-1-i} Y\left(\left(D_{z_{2}}^{(j-i)} z_{1}^{-\alpha_{0}-\mathcal{N}} z_{2}^{\alpha_{0}+\mathcal{N}} a\right)_{(j)} b, z_{2}\right),
$$

where $N$ is such that $\left(\mathcal{N}^{l} a\right)_{(j)} b=0$ for all $l \geq 0, j \geq N$.

Proof. By comparing the powers of $z_{1}$ in (5.10), we obtain

$$
P\left(a, b ; z_{1}, z_{2}\right)=\sum_{j=0}^{\infty} Y\left(\left(D_{z_{2}}^{(j)} \iota_{z_{1}, z_{2}} z_{12}^{-1} z_{1}^{-\alpha_{0}-\mathcal{N}} z_{2}^{\alpha_{0}+\mathcal{N}} a\right)_{(j)} b, z_{2}\right),
$$

using (2.6), (2.7) and (5.7). In this equation, the sum over $j$ goes only up to $j=N-1$. Then we apply the Leibniz rule and multiply by $z_{12}^{N}$ to finish the proof.

The next result is useful for constructing $\varphi$-twisted modules.

Proposition 5.7. Let $V$ be a vertex algebra, $\varphi$ an automorphism, $W$ a vector space, and $Y: V \rightarrow \operatorname{LFie}(W)$ a linear map satisfying the $\varphi$-equivariance (4.1) and the commutator formula (5.10) for $a \in V_{\alpha}, b \in V$. Then the logarithmic fields $Y(a, z)$ and $Y(b, z)$ are local, and the $n$-th product identity (4.2) holds for $a \in V_{\alpha}, b \in V$ and all $n \geq 0$.

Proof. As before, let $N \geq 0$ be such that $\left(\mathcal{N}^{l} a\right)_{(j)} b=0$ for all $l \geq 0$, $j \geq N$. Then in (5.10) the sum over $j$ goes only up to $j=N-1$. Using $z_{12} \delta_{\alpha+\mathcal{N}}\left(z_{1}, z_{2}\right)=0$, we derive from (5.10) the locality (3.1) of $a(z)=$ $Y(a, z)$ and $b(z)=Y(b, z)$.

By definition, their $n$-th product is 0 for $n \geq N$. To find it for $0 \leq n \leq$ $N-1$, we apply Proposition 3.6 and Lemma 5.6. Let us use the convention that $x^{(k)}=0$ for $k<0$. Then

$$
\begin{aligned}
& Y(a, z)_{(n)} Y(b, z) \\
& \quad=\left.D_{z_{1}}^{(N-1-n)} \sum_{i, j=0}^{N-1} z_{12}^{N-1-i} Y\left(\left(D_{z_{2}}^{(j-i)} z_{1}^{-\alpha_{0}-\mathcal{N}} z_{2}^{\alpha_{0}+\mathcal{N}_{a}} a\right){ }_{(j)} b, z_{2}\right)\right|_{z_{1}=z_{2}=z} .
\end{aligned}
$$


For a fixed $j$, we calculate using the Leibniz rule:

$$
\begin{aligned}
& \left.\sum_{i=0}^{N-1} D_{z_{1}}^{(N-1-n)}\left(z_{12}^{N-1-i}\left(D_{z_{2}}^{(j-i)} z_{1}^{-\alpha_{0}-\mathcal{N}} z_{2}^{\alpha_{0}+\mathcal{N}}\right)\right)\right|_{z_{1}=z_{2}=z} \\
& \quad=\left.\sum_{i=0}^{N-1} D_{z_{1}}^{(i-n)} D_{z_{2}}^{(j-i)}\left(z_{1}^{-\alpha_{0}-\mathcal{N}} z_{2}^{\alpha_{0}+\mathcal{N}}\right)\right|_{z_{1}=z_{2}=z} \\
& =D_{z}^{(j-n)}\left(z^{-\alpha_{0}-\mathcal{N}} z^{\alpha_{0}+\mathcal{N}}\right)=\delta_{j, n}
\end{aligned}
$$

Therefore, $Y(a, z)_{(n)} Y(b, z)=Y\left(a_{(n)} b, z\right)$.

As another application of Lemma 5.6, we obtain a formula relating the $(-1)$-st product with the normally ordered product given by Definition 3.5 (cf. [BK1, (3.13)]).

Lemma 5.8. In every $\varphi$-twisted $V$-module, we have

$$
: Y(a, z) Y(b, z):=\sum_{j=-1}^{N-1} z^{-j-1} Y\left(\left(\left(\begin{array}{c}
\alpha_{0}+\mathcal{N} \\
j+1
\end{array}\right) a\right)_{(j)} b, z\right)
$$

for $a \in V_{\alpha}$ and $b \in V$.

Proof. We proceed as in the proof of Proposition 5.7 for $n=-1$. We calculate for a fixed $0 \leq j \leq N-1$ :

$$
\begin{aligned}
& \left.\sum_{i=0}^{N-1} D_{z_{1}}^{(N)}\left(z_{12}^{N-1-i}\left(D_{z_{2}}^{(j-i)} z_{1}^{-\alpha_{0}-\mathcal{N}} z_{2}^{\alpha_{0}+\mathcal{N}}\right)\right)\right|_{z_{1}=z_{2}=z} \\
& \quad=\left.\sum_{i=0}^{j} D_{z_{1}}^{(i+1)} D_{z_{2}}^{(j-i)}\left(z_{1}^{-\alpha_{0}-\mathcal{N}} z_{2}^{\alpha_{0}+\mathcal{N}}\right)\right|_{z_{1}=z_{2}=z} \\
& \quad=D_{z}^{(j+1)}\left(z^{-\alpha_{0}-\mathcal{N}} z^{\alpha_{0}+\mathcal{N}}\right)-\left.D_{z_{1}}^{(0)} D_{z_{2}}^{(j+1)}\left(z_{1}^{-\alpha_{0}-\mathcal{N}} z_{2}^{\alpha_{0}+\mathcal{N}}\right)\right|_{z_{1}=z_{2}=z} \\
& \quad=-\left(\begin{array}{c}
\alpha_{0}+\mathcal{N} \\
j+1
\end{array}\right) z^{-j-1} .
\end{aligned}
$$

The rest of the proof follows again from Proposition 3.6 and Lemma 5.6.

5.4. Action of the Virasoro algebra. In this subsection, we assume that the vertex algebra $V$ is conformal, i.e., there exist $\omega \in V$ (conformal vector) and $c \in \mathbb{C}$ (central charge) such that (see, e.g., $\mathrm{K} 2$ ]):

$$
\omega_{(0)}=T, \quad \omega_{(1)} \omega=2 \omega, \quad \omega_{(2)} \omega=\frac{c}{2} \mathbf{1},
$$

and $\omega_{(j)} \omega=0$ for $j \geq 3$. Then the modes $L_{n}=\omega_{(n+1)}$ give a representation of the Virasoro Lie algebra on $V$ with a central charge $c$. In addition, it is usually assumed that the operator $L_{0}$ is semisimple on $V$.

Consider a (not necessarily locally finite) $\varphi \in \operatorname{Aut}(V)$ such that $\varphi(\omega)=\omega$, and a $\varphi$-twisted $V$-module $W$. Then the field $Y(\omega, z)$ on $W$ has only integral 
powers of $z$ and no $\zeta$, and its modes

$$
Y(\omega, z)=\sum_{n \in \mathbb{Z}} L_{n}^{W} z^{-n-2}, \quad L_{n}^{W} \in \operatorname{End}(W),
$$

give a representation of the Virasoro algebra on $W$ with the same central charge $c$.

Applying the commutator formula (5.11), we obtain:

$$
\begin{aligned}
& {\left[L_{-1}^{W}, Y(a, z)\right]=Y(T a, z)=D_{z} Y(a, z),} \\
& {\left[L_{0}^{W}, Y(a, z)\right]=z Y(T a, z)+Y\left(L_{0} a, z\right), \quad a \in V .}
\end{aligned}
$$

Then from $z D_{z}=D_{\zeta}$, we have

$$
\left[L_{0}^{W}, Y(a, z)\right]=\left(D_{\zeta}+\Delta\right) Y(a, z), \quad \text { if } \quad L_{0} a=\Delta a .
$$

Remark 5.9. Assume that $L_{0}$ is semisimple on $V$, but $\varphi$ is not semisimple. Then $L_{0}^{W}$ is not semisimple on $W$. Thus, $W$ is a (untwisted) $V^{\varphi}$-module with a non-semisimple action of $L_{0}^{W}$, also known as a logarithmic module (see $[\mathrm{AM}]$ ).

Lemma 5.10. Let $W$ be a $\varphi$-twisted $V$-module. Assume that the operator $L_{0}$ is semisimple on $V$ with integral eigenvalues, and the operator $e^{2 \pi \mathrm{i} L_{0}^{W}}$ is well defined on $W$. Then

$$
e^{2 \pi \mathrm{i} L_{0}^{W}} Y(a, z) e^{-2 \pi \mathrm{i} L_{0}^{W}}=e^{2 \pi \mathrm{i} D_{\zeta}} Y(a, z)=Y(\varphi a, z)
$$

when acting on $W$, for every $a \in V$.

Proof. Indeed,

$$
e^{2 \pi \mathrm{i} L_{0}^{W}} Y(a, z) e^{-2 \pi \mathrm{i} L_{0}^{W}}=e^{2 \pi \mathrm{i} \operatorname{ad}\left(L_{0}^{W}\right)} Y(a, z)=e^{2 \pi \mathrm{i}\left(D_{\zeta}+\Delta\right)} Y(a, z)
$$

when $L_{0} a=\Delta a$.

Lemma 5.10 can be used to define $e^{2 \pi \mathrm{i} L_{0}^{W}}$ on the whole $W$, provided it can be defined on a set of generators of $W$ as a $\varphi$-twisted $V$-module; in particular, when these generators are eigenvectors of $L_{0}^{W}$. This can be used to define a grading of $W$ as in [H, Definition 3.1].

\section{Twisted modules of Affine And Heisenberg vertex Algebras}

In this section, we describe all twisted modules of affine and Heisenberg vertex algebras in terms of modules over certain twisted versions of the corresponding Lie algebras. We also determine the action of the Virasoro algebra. For the Heisenberg vertex algebra, all twisted irreducible highestweight modules are constructed explicitly. 
6.1. Universal affine vertex algebras. Let us first recall the definition of affine Lie algebras, following [K1. Consider a finite-dimensional Lie algebra $\mathfrak{g}$ equipped with a nondegenerate symmetric invariant bilinear form $(\cdot \mid \cdot)$, normalized so that the square length of a long root is 2 in the case when $\mathfrak{g}$ is simple. The affine Lie algebra $\hat{\mathfrak{g}}=\mathfrak{g}\left[t, t^{-1}\right] \oplus \mathbb{C} K$ has the Lie brackets

$$
\left[a t^{m}, b t^{n}\right]=[a, b] t^{m+n}+m \delta_{m,-n}(a \mid b) K, \quad\left[K, a t^{m}\right]=0 .
$$

For a fixed $\kappa \in \mathbb{C}$, called the level, the (generalized) Verma module $M\left(\kappa \Lambda_{0}\right)=$ $\operatorname{Ind}_{\mathfrak{g}[t] \oplus \mathbb{C} K}^{\hat{\mathfrak{g}}} \mathbb{C}$ is defined by letting $\mathfrak{g}[t]$ act trivially on $\mathbb{C}$ and $K$ act as $\kappa$. Then $M\left(\kappa \Lambda_{0}\right)$ is a highest-weight $\hat{\mathfrak{g}}$-module with a highest-weight vector the image of $1 \in \mathbb{C}$, which will be denoted 1 . Notice that as a vector space, $M\left(\kappa \Lambda_{0}\right) \cong U\left(\mathfrak{g}\left[t^{-1}\right] t^{-1}\right)$.

Due to FZ, $M\left(\kappa \Lambda_{0}\right)$ has the structure of a vertex algebra, which is called the universal affine vertex algebra at level $\kappa$ and is denoted $V^{\kappa}(\mathfrak{g})$. It has a vacuum vector $\mathbf{1}$ and is generated by the local fields

$$
Y\left(\left(a t^{-1}\right) \mathbf{1}, z\right)=\sum_{m \in \mathbb{Z}}\left(a t^{m}\right) z^{-m-1}, \quad a \in \mathfrak{g}
$$

(see, e.g., $\underline{\mathrm{K} 2}$ for more details). For simplicity of notation, let us identify $a \in \mathfrak{g}$ with $\left(a t^{-1}\right) \mathbf{1} \in V^{\kappa}(\mathfrak{g})$; then $a_{(m)}=a t^{m}$ as operators on $V^{\kappa}(\mathfrak{g})$. By the commutator formula (2.4), the Lie brackets (6.1) are equivalent to the relations

$$
a_{(0)} b=[a, b], \quad a_{(1)} b=(a \mid b) \kappa \mathbf{1}, \quad a_{(j)} b=0 \quad(j \geq 2)
$$

for $a, b \in \mathfrak{g}$.

Now suppose that $\mathfrak{g}$ is simple or abelian, and let $h^{\vee}$ be the dual Coxeter number of $\mathfrak{g}$ in the case when it is simple. When $\mathfrak{g}$ is abelian, we set $h^{\vee}=0$. Then the vertex algebra $V^{\kappa}(\mathfrak{g})$ is conformal for $\kappa \neq-h^{\vee}$. Pick dual bases $\left\{v_{i}\right\}$ and $\left\{v^{i}\right\}$ for $\mathfrak{g}$ with respect to $(\cdot \mid \cdot)$. The conformal vector $\omega \in V^{\kappa}(\mathfrak{g})$ is given by the Sugawara construction

$$
\omega=\frac{1}{2\left(\kappa+h^{\vee}\right)} \sum_{i=1}^{\operatorname{dim} \mathfrak{g}} v_{(-1)}^{i} v_{i}, \quad \kappa \neq-h^{\vee}
$$

(see, e.g., [K2]). The Virasoro central charge is $c=\kappa \operatorname{dim} \mathfrak{g} /\left(\kappa+h^{\vee}\right)$. The operator $L_{0}$ satisfies $L_{0} \mathbf{1}=0, L_{0} a=a(a \in \mathfrak{g})$, and it defines a $\mathbb{Z}_{+}$-grading of $V^{\kappa}(\mathfrak{g})$ by its eigenvalues.

6.2. $\varphi$-twisted modules of $V^{\kappa}(\mathfrak{g})$. From now on, $\varphi$ will be an automorphism of $\mathfrak{g}$ such that $(\cdot \mid \cdot)$ is $\varphi$-invariant. Then $\varphi$ induces automorphisms of $\hat{\mathfrak{g}}$ and $V=V^{\kappa}(\mathfrak{g})$, which we will again denote as $\varphi$. Notice that $\varphi(\omega)=\omega$. Since the eigenspaces of $L_{0}$ in $V$ are finite-dimensional and $\varphi$-invariant, $\varphi$ is locally finite on $V$.

Writing again $\varphi=\sigma e^{-2 \pi \mathrm{i} \mathcal{N}}$, we have $\sigma \in \operatorname{Aut}(\mathfrak{g}), \mathcal{N} \in \operatorname{Der}(\mathfrak{g})$, and

$$
(\sigma a \mid \sigma b)=(a \mid b), \quad(\mathcal{N} a \mid b)+(a \mid \mathcal{N} b)=0 .
$$


As before, we denote the eigenspaces of $\sigma$ by

$$
\mathfrak{g}_{\alpha}=\left\{a \in \mathfrak{g} \mid \sigma a=e^{-2 \pi \mathrm{i} \alpha} a\right\}, \quad \alpha \in \mathbb{C} / \mathbb{Z} .
$$

If $W$ is a $\varphi$-twisted $V$-module, then by (5.9) and (6.2), we have:

$$
\left[a_{(m+\mathcal{N})}, b_{(k+\mathcal{N})}\right]=[a, b]_{(m+k+\mathcal{N})}+\delta_{m,-n}((m+\mathcal{N}) a \mid b) \kappa I
$$

for $a \in \mathfrak{g}_{\alpha}, b \in \mathfrak{g}_{\beta}, m \in \alpha, k \in \beta$. Hence, the modes $a_{(m+\mathcal{N})}$ close a Lie algebra, which can be described as follows (cf. [K1, Chapter 8]).

Let $\tilde{\mathfrak{g}}=\bigoplus_{\alpha \in \mathbb{C} / \mathbb{Z}} \mathfrak{g}[t] t^{\alpha}$ be the loop algebra, whose elements are finite sums of $a t^{m}(a \in \mathfrak{g}, m \in \mathbb{C})$, with the Lie bracket $\left[a t^{m}, b t^{n}\right]=[a, b] t^{m+n}$. We define an automorphism $\tilde{\sigma}$ of $\tilde{\mathfrak{g}}$ by $\tilde{\sigma}\left(a t^{m}\right)=e^{2 \pi \mathrm{i} m} \sigma(a) t^{m}$. The subalgebra $\tilde{\mathfrak{g}}_{\sigma}$ of fixed points under $\tilde{\sigma}$ is spanned by $a t^{m}\left(a \in \mathfrak{g}_{\alpha}, m \in \alpha\right)$. The loop algebra $\tilde{\mathfrak{g}}$ has a 2 -cocycle $\gamma$ given by

$$
\gamma\left(a t^{m}, b t^{n}\right)=m \delta_{m,-n}(a \mid b)=\operatorname{Res}_{t}\left(\partial_{t}\left(a t^{m}\right) \mid b t^{n}\right),
$$

which gives rise to a central extension of $\tilde{\mathfrak{g}}$ similar to $\hat{\mathfrak{g}}$ (see (6.1)). When restricted to $\tilde{\mathfrak{g}}_{\sigma}$, we obtain the Lie algebra $\hat{\mathfrak{g}}_{\sigma}=\tilde{\mathfrak{g}}_{\sigma} \oplus \mathbb{C} K$. It is easy to check that

$$
\gamma_{\mathcal{N}}\left(a t^{m}, b t^{n}\right)=\delta_{m,-n}((m+\mathcal{N}) a \mid b)=\operatorname{Res}_{t}\left(\left(\partial_{t}+t^{-1} \mathcal{N}\right)\left(a t^{m}\right) \mid b t^{n}\right)
$$

again defines a 2-cocycle on $\tilde{\mathfrak{g}}$. If we use $\gamma_{\mathcal{N}}$ instead of $\gamma$, we obtain the Lie algebra $\hat{\mathfrak{g}}_{\varphi}=\tilde{\mathfrak{g}}_{\sigma} \oplus \mathbb{C} K$.

Definition 6.1. The $\varphi$-twisted affinization of $\mathfrak{g}$ is the Lie algebra $\hat{\mathfrak{g}}_{\varphi}$ spanned by a central element $K$ and elements $a t^{m}\left(a \in \mathfrak{g}_{\alpha}, m \in \alpha\right)$, with the Lie bracket

$$
\left[a t^{m}, b t^{n}\right]=[a, b] t^{m+n}+\delta_{m,-n}((m+\mathcal{N}) a \mid b) K .
$$

Proposition 6.2. When the Lie algebra $\mathfrak{g}$ is simple, there exists a Dynkin diagram automorphism $\mu$ of $\mathfrak{g}$, such that $\hat{\mathfrak{g}}_{\varphi} \cong \hat{\mathfrak{g}}_{\mu}$ is a (possibly twisted) affine Kac-Moody algebra.

Proof. We have $\mathcal{N}=\operatorname{ad}_{y}$ for some $y \in \mathfrak{g}$. Then

$$
\operatorname{Res}_{t}\left(t^{-1} \mathcal{N}\left(a t^{m}\right) \mid b t^{n}\right)=\operatorname{Res}_{t}\left(y t^{-1} \mid\left[a t^{m}, b t^{n}\right]\right),
$$

and the cocycle $\gamma_{\mathcal{N}}$ is equivalent to $\gamma$. Hence, $\hat{\mathfrak{g}}_{\varphi} \cong \hat{\mathfrak{g}}_{\sigma}$.

We can write $\sigma=\mu e^{2 \pi \mathrm{iad}_{x}}$ for some semisimple $x \in \mathfrak{g}$ and a Dynkin diagram automorphism $\mu$, so that $\mu$ commutes with $\operatorname{ad}_{x}$. Then the map $t^{\mathrm{ad}_{x}}$, defined by $t^{\operatorname{ad}_{x}}\left(a t^{m}\right)=a t^{m+p}$ whenever $[x, a]=p a$, is an isomorphism from $\tilde{\mathfrak{g}}_{\sigma}$ to $\tilde{\mathfrak{g}}_{\mu}$ (cf. [K1, Proposition 8.5]). It lifts to an isomorphism $\hat{\mathfrak{g}}_{\sigma} \cong \hat{\mathfrak{g}}_{\mu}$, since the cocycle

$$
\operatorname{Res}_{t}\left(\partial_{t}\left(t^{\operatorname{ad}_{x}} a t^{m}\right) \mid t^{\operatorname{ad}_{x}} b t^{n}\right)=\operatorname{Res}_{t}\left(\left(\partial_{t}+t^{-1} \operatorname{ad}_{x}\right)\left(a t^{m}\right) \mid b t^{n}\right)=\gamma_{\mathrm{ad}_{x}}\left(a t^{m} \mid b t^{n}\right)
$$

is equivalent to $\gamma$. Finally, $\hat{\mathfrak{g}}_{\mu}$ is an affine Kac-Moody algebra by K1, Theorem 8.3]. 
A $\hat{\mathfrak{g}}_{\varphi}$-module $W$ is called restricted if for every $a \in \mathfrak{g}_{\alpha}, m \in \alpha, v \in W$, there is an integer $L$ such that $\left(a t^{m+i}\right) v=0$ for all $i \in \mathbb{Z}, i \geq L$. For example, every highest-weight $\hat{\mathfrak{g}}_{\varphi}$-module is restricted (see [K1]). We say that $W$ has level $\kappa$ if $K$ acts on it as $\kappa I$. Then we have the following correspondence of modules (cf. [Li2, KRR]).

Theorem 6.3. Every $\varphi$-twisted $V^{\kappa}(\mathfrak{g})$-module is a restricted $\hat{\mathfrak{g}}_{\varphi}$-module of level $\kappa$ and, conversely, every restricted $\hat{\mathfrak{g}}_{\varphi}$-module of level $\kappa$ uniquely extends to a $\varphi$-twisted $V^{\kappa}(\mathfrak{g})$-module.

Proof. In one direction the statement is obvious from the definitions. Conversely, suppose that $W$ is a restricted $\hat{\mathfrak{g}}_{\varphi}$-module of level $\kappa$. For $a \in \mathfrak{g}_{\alpha}$, we define the logarithmic field $Y(a, z) \in \operatorname{LFie}_{\alpha}(W)$ by

$$
Y(a, z)=\sum_{m \in \alpha} z^{-m-1}\left(\left(e^{-\zeta \mathcal{N}} a\right) t^{m}\right) .
$$

Then (4.4) holds, which implies the $\varphi$-equivariance (4.1). By (5.2), the modes of $a$ are $a_{(m+\mathcal{N})}=a t^{m}$.

Comparing (6.5) and (6.6), we see that the commutator formula (5.10) holds for $a \in \mathfrak{g}_{\alpha}, b \in \mathfrak{g}_{\beta}$. By Proposition 5.7, the fields $Y(a, z)$ are local and satisfy the $n$-th product identity (4.2) for $n \geq 0$. Let $\mathcal{W}$ be the local collection $\{Y(a, z)\}_{a \in \mathfrak{g}}$, and $\overline{\mathcal{W}} \subset \operatorname{LFie}(W)$ be the vertex algebra generated by it (see Theorem [3.7). Since $V^{\kappa}(\mathfrak{g}) \cong U\left(\mathfrak{g}\left[t^{-1}\right] t^{-1}\right)$, the map $Y$ can be extended uniquely to a vertex algebra homomorphism from $V^{\kappa}(\mathfrak{g})$ to $\overline{\mathcal{W}}$. This endows $W$ with the structure of a $\varphi$-twisted $V^{\kappa}(\mathfrak{g})$-module.

As in Section 5.4, the modes of $Y(\omega, z)$ give a representation of the Virasoro Lie algebra on every $\varphi$-twisted $V^{\kappa}(\mathfrak{g})$-module $W$. To state the explicit formula, let us define a linear operator $\mathcal{S}$ on $\mathfrak{g}$ by $\mathcal{S} a=\alpha_{0} a$ for $a \in \mathfrak{g}_{\alpha}$, $\alpha \in \mathbb{C} / \mathbb{Z}$ and $\alpha_{0} \in \alpha$ such that $-1<\operatorname{Re} \alpha_{0} \leq 0$. Recall that the normally ordered product of two logarithmic fields is given by Definition 3.5.

Lemma 6.4. In every $\varphi$-twisted $V^{\kappa}(\mathfrak{g})$-module $W$, we have

$$
2\left(\kappa+h^{\vee}\right) Y(\omega, z)=\sum_{i=1}^{\operatorname{dim} \mathfrak{g}}: X\left(v^{i}, z\right) X\left(v_{i}, z\right):-z^{-1} X(\bar{\omega}, z)-z^{-2} \kappa \operatorname{tr}\left(\begin{array}{l}
\mathcal{S} \\
2
\end{array}\right) I,
$$

where

$$
\bar{\omega}=\sum_{i=1}^{\operatorname{dim} \mathfrak{g}}\left[(\mathcal{S}+\mathcal{N}) v^{i}, v_{i}\right]
$$

using the notation from (6.3).

Proof. Applying Lemma 5.8 and (6.2), we obtain for $a, b \in \mathfrak{g}$ :

$$
\begin{aligned}
Y\left(a_{(-1)} b, z\right) & =: Y(a, z) Y(b, z): \\
& -z^{-1} Y([(\mathcal{S}+\mathcal{N}) a, b], z)-z^{-2}\left(\left(\begin{array}{c}
\mathcal{S}+\mathcal{N} \\
2
\end{array}\right) a \mid b\right) \kappa I .
\end{aligned}
$$


Then we use this with (6.3) to find $Y(\omega, z)$. Note that $Y(\omega, z)$ is independent of $\zeta$, because $\varphi \omega=\omega$. Hence, we can set $\zeta=0$ and replace $Y$ with $X$ (see Lemma 4.91). Finally,

$$
\sum_{i=1}^{\operatorname{dim} \mathfrak{g}}\left(\left(\begin{array}{c}
\mathcal{S}+\mathcal{N} \\
2
\end{array}\right) v^{i} \mid v_{i}\right)=\operatorname{tr}\left(\begin{array}{c}
\mathcal{S}+\mathcal{N} \\
2
\end{array}\right)=\operatorname{tr}\left(\begin{array}{l}
\mathcal{S} \\
2
\end{array}\right)
$$

since we can find a basis for $\mathfrak{g}$ in which $\mathcal{S}$ is diagonal and $\mathcal{N}$ is strictly upper triangular.

Note that we can pick the dual bases for $\mathfrak{g}$ so that $v^{i} \in \mathfrak{g}_{\alpha^{i}}$ and $v_{i} \in \mathfrak{g}_{-\alpha^{i}}$ for some $\alpha^{i} \in \mathbb{C} / \mathbb{Z}$. Moreover, $\bar{\omega} \in \mathfrak{g}_{0}$ since $\varphi \bar{\omega}=\bar{\omega}$. Then from Lemma 6.4, we obtain for the modes (5.12):

$$
2\left(\kappa+h^{\vee}\right) L_{n}^{W}=\sum_{i=1}^{\operatorname{dim} \mathfrak{g}} \sum_{m \in \alpha^{i}}:\left(v^{i} t^{m}\right)\left(v_{i} t^{n-m}\right):-\bar{\omega} t^{n}-\delta_{n, 0} \kappa \operatorname{tr}\left(\begin{array}{l}
\mathcal{S} \\
2
\end{array}\right) I,
$$

for any $n \in \mathbb{Z}$.

6.3. $\varphi$-twisted modules of the Heisenberg vertex algebra. Now assume that $\mathfrak{g}$ is abelian, and denote it by $\mathfrak{h}$ instead of $\mathfrak{g}$. The affine Lie algebra $\hat{\mathfrak{h}}$ is called the Heisenberg Lie algebra and its irreducible highestweight module $\mathcal{F}=M\left(\Lambda_{0}\right)=V^{1}(\mathfrak{h})$ is known as the (bosonic) Fock space or the Heisenberg vertex algebra. Explicitly, the Lie bracket in $\hat{\mathfrak{h}}$ is given by

$$
\left[a t^{m}, b t^{n}\right]=\delta_{m,-n}((m+\mathcal{N}) a \mid b) K .
$$

Note that $V^{\kappa}(\mathfrak{h}) \cong V^{1}(\mathfrak{h})$ for any $\kappa \neq 0$, so we can assume $\kappa=1$ without loss of generality.

Let us split $\mathbb{C}$ as a disjoint union of subsets $\mathbb{C}^{+}, \mathbb{C}^{-}=-\mathbb{C}^{+}$and $\{0\}$. We will take

$$
\mathbb{C}^{+}=\{\gamma \in \mathbb{C} \mid \operatorname{Re} \gamma>0\} \cup\{\gamma \in \mathbb{C} \mid \operatorname{Re} \gamma=0, \operatorname{Im} \gamma>0\} .
$$

Then the $\varphi$-twisted affinization $\hat{\mathfrak{h}}_{\varphi}$ has a triangular decomposition $\hat{\mathfrak{h}}_{\varphi}=$ $\hat{\mathfrak{h}}_{\varphi}^{-} \oplus \hat{\mathfrak{h}}_{\varphi}^{0} \oplus \hat{\mathfrak{h}}_{\varphi}^{+}$(direct sum of vector spaces), where

$$
\hat{\mathfrak{h}}_{\varphi}^{ \pm}=\operatorname{span}\left\{a t^{m} \mid a \in \mathfrak{h}_{\alpha}, \alpha \in \mathbb{C} / \mathbb{Z}, m \in \alpha \cap \mathbb{C}^{ \pm}\right\}
$$

and

$$
\hat{\mathfrak{h}}_{\varphi}^{0}=\operatorname{span}\left\{a t^{0} \mid a \in \mathfrak{h}_{0}\right\} \oplus \mathbb{C} K .
$$

It is clear from (6.9) that $\hat{\mathfrak{h}}_{\varphi}^{ \pm}$are abelian subalgebras of $\hat{\mathfrak{h}}_{\varphi}$, and $\hat{\mathfrak{h}}_{\varphi}^{0}$ is a finite-dimensional subalgebra satisfying $\left[\hat{\mathfrak{h}}_{\varphi}^{0}, \hat{\mathfrak{h}}_{\varphi}^{0}\right] \subset \mathbb{C} K,\left[\hat{\mathfrak{h}}_{\varphi}^{0}, \hat{\mathfrak{h}}_{\varphi}^{ \pm}\right]=\{0\}$.

Let $W$ be an $\hat{\mathfrak{h}}_{\varphi}$-module. A highest-weight vector (also called a vacuum vector) in $W$ is $v \in W$ such that $\hat{\mathfrak{h}}_{\varphi}^{+} v=0$. All such vectors form an $\hat{\mathfrak{h}}_{\varphi^{-}}^{0}$ submodule $R$ of $W$. If $W$ is generated by $R$ as an $\hat{\mathfrak{h}}_{\varphi}$-module, we say that $W$ is a highest-weight module. As usual, examples can be constructed as 
induced modules. Starting from any $\hat{\mathfrak{h}}_{\varphi}^{0}$-module $R$ such that $K=I$, we define the (generalized) Verma module

$$
M_{\varphi}(R)=\operatorname{Ind}_{\hat{\mathfrak{h}}_{\varphi}^{+} \oplus \hat{\mathfrak{h}}_{\varphi}^{0}}^{\hat{\mathfrak{h}}_{\varphi}} R \cong S\left(\hat{\mathfrak{h}}_{\varphi}^{-}\right) \otimes_{\mathbb{C}} R
$$

where $\hat{\mathfrak{h}}_{\varphi}^{+}$acts trivially on $R$. It is a standard fact that the $\hat{\mathfrak{h}}_{\varphi}$-module $M_{\varphi}(R)$ is irreducible for any irreducible $\hat{\mathfrak{h}}_{\varphi}^{0}$-module $R$ (cf. [FLM, KRR]). Therefore, all irreducible highest-weight $\hat{\mathfrak{h}}_{\varphi}$-modules have this form. In addition, all of them are restricted, so they give rise to $\varphi$-twisted $\mathcal{F}$-modules.

We will present two explicit examples of linear operators $\sigma$ and $\mathcal{N}$ on $\mathfrak{h}$ satisfying (6.4). Fix a positive integer $\ell$ and $\alpha_{0} \in \mathbb{C}$ such that $-1<\operatorname{Re} \alpha_{0} \leq$ 0 , and set $\lambda=e^{-2 \pi \mathrm{i} \alpha_{0}}$.

Example 6.5 $(\operatorname{dim} \mathfrak{h}=2 \ell)$. Consider a vector space $\mathfrak{h}$ with a basis $\left\{v_{1}, \ldots, v_{2 \ell}\right\}$ such that $\left(v_{i} \mid v_{j}\right)=\delta_{i+j, 2 \ell+1}$ and

$$
\sigma v_{i}=\left\{\begin{array}{l}
\lambda v_{i}, \quad 1 \leq i \leq \ell, \\
\lambda^{-1} v_{i}, \ell+1 \leq i \leq 2 \ell,
\end{array} \quad \mathcal{N} v_{i}=\left\{\begin{array}{l}
v_{i+1}, \quad 1 \leq i \leq \ell-1, \\
-v_{i+1}, \ell+1 \leq i \leq 2 \ell-1, \\
0, \quad i=\ell, 2 \ell
\end{array}\right.\right.
$$

Due to the symmetry $v_{i} \mapsto(-1)^{i} v_{\ell+i}, v_{\ell+i} \mapsto(-1)^{i+\ell+1} v_{i}(1 \leq i \leq \ell)$, we can assume that $\alpha_{0} \in \mathbb{C}^{-} \cup\{0\}$.

Example 6.6 $(\operatorname{dim} \mathfrak{h}=2 \ell-1)$. Here $\lambda= \pm 1$, so $\alpha_{0}=0$ or $-1 / 2$. Define $\mathfrak{h}$ as a vector space with a basis $\left\{v_{1}, \ldots, v_{2 \ell-1}\right\}$ such that $\left(v_{i} \mid v_{j}\right)=\delta_{i+j, 2 \ell}$ and

$$
\sigma v_{i}=\lambda v_{i}, \quad 1 \leq i \leq 2 \ell-1, \quad \mathcal{N} v_{i}= \begin{cases}(-1)^{i+1} v_{i+1}, & 1 \leq i \leq 2 \ell-2 \\ 0, & i=2 \ell-1\end{cases}
$$

Proposition 6.7. Let $\mathfrak{h}$ be a finite-dimensional vector space, equipped with a nondegenerate symmetric bilinear form $(\cdot \mid \cdot)$ and with commuting linear operators $\sigma, \mathcal{N}$ satisfying (6.4), such that $\sigma$ is invertible and semisimple and $\mathcal{N}$ is nilpotent. Then $\mathfrak{h}$ is an orthogonal direct sum of subspaces that are like Examples 6.5 and 6.6.

Proof. This follows from the well-known classification, up to conjugation, of orthogonal and skew-symmetric matrices over $\mathbb{C}$ (see [Ga, $\mathrm{HM}]$ ).

In the next two subsections, we will consider separately the above two examples. In each case, we will describe explicitly the $\varphi$-twisted affinization $\hat{\mathfrak{h}}_{\varphi}$ and its irreducible highest-weight modules $M_{\varphi}(R)$. We will also determine the action of the Virasoro algebra using (6.8). Note that in (6.8), we have $\bar{\omega}=0$ and the normally ordered product is needed only for $n=0$, since $\mathfrak{h}$ is abelian. 
6.4. The case $\operatorname{dim} \mathfrak{h}=2 \ell$. First, let $\mathfrak{h}$ be as in Example 6.5. Then $\hat{\mathfrak{h}}_{\varphi}$ is the Lie algebra spanned by a central element $K$ and elements $v_{i} t^{\alpha_{0}+n}$, $v_{\ell+i} t^{-\alpha_{0}+n}(1 \leq i \leq \ell, n \in \mathbb{Z})$, with Lie brackets given by (6.9). More explicitly, for $1 \leq i \leq \ell$ and $1 \leq j \leq 2 \ell$, we have:

$$
\begin{aligned}
{\left[v_{i} t^{m}, v_{j} t^{k}\right] } & =m \delta_{m+k, 0} \delta_{i+j, 2 \ell+1} K+\delta_{m+k, 0}\left(1-\delta_{i, \ell}\right) \delta_{i+j, 2 \ell} K, \\
{\left[v_{\ell+i} t^{m}, v_{j} t^{k}\right] } & =m \delta_{m+k, 0} \delta_{i+j, \ell+1} K-\delta_{m+k, 0}\left(1-\delta_{i, \ell}\right) \delta_{i+j, \ell} K .
\end{aligned}
$$

It follows from (5.2) that for $1 \leq j \leq \ell$ :

$$
\begin{aligned}
Y\left(v_{j}, z\right) & =\sum_{i=j}^{\ell} \sum_{m \in \alpha_{0}+\mathbb{Z}}(-\zeta)^{(i-j)}\left(v_{i} t^{m}\right) z^{-m-1}, \\
Y\left(v_{\ell+j}, z\right) & =\sum_{i=j}^{\ell} \sum_{m \in-\alpha_{0}+\mathbb{Z}} \zeta^{(i-j)}\left(v_{\ell+i} t^{m}\right) z^{-m-1} .
\end{aligned}
$$

The action of the Virasoro algebra is determined by (6.8) with $\bar{\omega}=0$. The dual basis $\left\{v^{i}\right\}$ to the basis $\left\{v_{i}\right\}$ is given by $v^{i}=v_{2 \ell+1-i}$. As we already pointed out, the normally ordered product in (6.8) is needed only for $L_{0}^{W}$. Therefore,

$$
L_{k}^{W}=\sum_{i=1}^{\ell} \sum_{n \in \mathbb{Z}}\left(v_{i} t^{\alpha_{0}+n+k}\right)\left(v_{2 \ell+1-i} t^{-\alpha_{0}-n}\right), \quad k \neq 0 .
$$

The triangular decomposition of $\hat{\mathfrak{h}}_{\varphi}$ depends on whether $\alpha_{0} \in \mathbb{C}^{-}$or $\alpha_{0}=0$. Suppose first that $\alpha_{0} \in \mathbb{C}^{-}$. Then $\hat{\mathfrak{h}}_{\varphi}^{0}=\mathbb{C} K$ and $R=\mathbb{C}$ with $K=I$ acting as the identity operator. We have:

$$
M_{\varphi}(R) \cong \mathbb{C}\left[x_{i, 0}, x_{j, n}\right]_{1 \leq i \leq \ell, 1 \leq j \leq 2 \ell, n=1,2,3, \ldots},
$$

where for $1 \leq i \leq \ell, n=0,1,2, \ldots$,

$$
v_{i} t^{\alpha_{0}-n}=x_{i, n}, \quad v_{\ell+i} t^{-\alpha_{0}-n-1}=x_{\ell+i, n+1},
$$

and

$$
\begin{aligned}
v_{i} t^{\alpha_{0}+n+1} & =\left(\alpha_{0}+n+1\right) \partial_{x_{2 \ell+1-i, n+1}}+\left(1-\delta_{i, \ell}\right) \partial_{x_{2 \ell-i, n+1}}, \\
v_{\ell+i} t^{-\alpha_{0}+n} & =\left(-\alpha_{0}+n\right) \partial_{x_{\ell+1-i, n}}-\left(1-\delta_{i, \ell}\right) \partial_{x_{\ell-i, n}} .
\end{aligned}
$$

Lemma 6.8. For $\alpha_{0} \in \mathbb{C}^{-}$and $W=M_{\varphi}(R)$ as in (6.11), we have

$$
\begin{aligned}
L_{0}^{W} & =\sum_{i=1}^{\ell} \sum_{n=0}^{\infty} x_{i, n}\left(\left(-\alpha_{0}+n\right) \partial_{x_{i, n}}-\left(1-\delta_{i, 1}\right) \partial_{x_{i-1, n}}\right) \\
& +\sum_{i=1}^{\ell} \sum_{n=1}^{\infty} x_{\ell+i, n}\left(\left(\alpha_{0}+n\right) \partial_{x_{\ell+i, n}}+\left(1-\delta_{i, 1}\right) \partial_{x_{\ell+i-1, n}}\right) \\
& -\frac{\ell}{2}\left(\alpha_{0}^{2}+\alpha_{0}\right) I .
\end{aligned}
$$


Proof. Let us apply (6.8) with $v^{i}=v_{2 \ell+1-i}$. We observe that there are only two cases in which the normally ordered product from Definition 3.5 differs from the one obtained by placing all $x_{i, n}$ to the left of all $\partial_{x_{i, n}}$. First, for $\ell+1 \leq i \leq 2 \ell$

$$
\begin{aligned}
& :\left(v^{i} t^{\alpha_{0}}\right)\left(v_{i} t^{-\alpha_{0}}\right):=\left(v_{i} t^{-\alpha_{0}}\right)\left(v^{i} t^{\alpha_{0}}\right) \\
& \quad=x_{2 \ell+1-i, 0}\left(\left(-\alpha_{0}\right) \partial_{x_{2 \ell+1-i, 0}}-\left(1-\delta_{i, 2 \ell}\right) \partial_{x_{2 \ell-i, 0}}\right)-\alpha_{0} I .
\end{aligned}
$$

Second, for $\operatorname{Re} \alpha_{0}<0$ and $1 \leq i \leq \ell$,

$$
\begin{aligned}
& :\left(v^{i} t^{-\alpha_{0}-1}\right)\left(v_{i} t^{\alpha_{0}+1}\right):=\left(v_{i} t^{\alpha_{0}+1}\right)\left(v^{i} t^{-\alpha_{0}-1}\right) \\
& \quad=x_{2 \ell+1-i, 1}\left(\left(\alpha_{0}+1\right) \partial_{x_{2 \ell+1-i, 1}}+\left(1-\delta_{i, \ell}\right) \partial_{x_{2 \ell-i, 1}}\right)+\left(\alpha_{0}+1\right) I .
\end{aligned}
$$

On the other hand, when $\operatorname{Re} \alpha_{0}<0$, we have

$$
\mathcal{S} v_{i}=\alpha_{0} v_{i}, \quad \mathcal{S} v_{\ell+i}=\left(-\alpha_{0}-1\right) v_{\ell+i}, \quad 1 \leq i \leq \ell,
$$

from where we find $\operatorname{tr}\left(\begin{array}{l}\mathcal{S} \\ 2\end{array}\right)=\ell\left(\alpha_{0}^{2}+\alpha_{0}+1\right)$. When $\operatorname{Re} \alpha_{0}=0$, we have

$$
\mathcal{S} v_{i}=\alpha_{0} v_{i}, \quad \mathcal{S} v_{\ell+i}=-\alpha_{0} v_{\ell+i}, \quad 1 \leq i \leq \ell,
$$

which gives $\operatorname{tr}\left(\begin{array}{l}\mathcal{S} \\ 2\end{array}\right)=\ell \alpha_{0}^{2}$. In both cases, the combined contribution from the difference of the normally ordered products and $\operatorname{tr}\left(\begin{array}{l}\mathcal{S} \\ 2\end{array}\right)$ is $-\frac{\ell}{2}\left(\alpha_{0}^{2}+\alpha_{0}\right)$.

Now consider the case when $\alpha_{0}=0$ in Example 6.5. Then $\hat{\mathfrak{h}}_{\varphi}^{0}=\mathfrak{h} t^{0} \oplus \mathbb{C} K$ is a direct sum of a finite-dimensional Heisenberg Lie algebra and the central ideal span $\left\{v_{\ell} t^{0}, v_{2 \ell} t^{0}\right\}$. We have the following irreducible $\hat{\mathfrak{h}}_{\varphi}^{0}$-modules $R$ with $K=I$ :

$$
R_{a_{1}, a_{2}}=\mathbb{C}\left[x_{i, 0}\right]_{1 \leq i \leq \ell-1} \quad\left(a_{1}, a_{2} \in \mathbb{C}\right),
$$

where for $1 \leq i \leq \ell-1$,

$$
v_{i} t^{0}=x_{i, 0}, \quad v_{\ell+i} t^{0}=-\partial_{x_{\ell-i, 0}}, \quad v_{\ell} t^{0}=a_{1} I, \quad v_{2 \ell} t^{0}=a_{2} I .
$$

Then

$$
M_{\varphi}\left(R_{a_{1}, a_{2}}\right) \cong \mathbb{C}\left[x_{i, 0}, x_{j, n}\right]_{1 \leq i \leq \ell-1,1 \leq j \leq 2 \ell, n=1,2,3, \ldots},
$$

where for $1 \leq i \leq \ell$ and $n=1,2,3, \ldots$,

$$
\begin{aligned}
v_{i} t^{-n} & =x_{i, n}, \quad v_{\ell+i} t^{-n}=x_{\ell+i, n}, \\
v_{i} t^{n} & =n \partial_{x_{2 \ell+1-i, n}}+\left(1-\delta_{i, \ell}\right) \partial_{x_{2 \ell-i, n}}, \\
v_{\ell+i} t^{n} & =n \partial_{x_{\ell+1-i, n}}-\left(1-\delta_{i, \ell}\right) \partial_{x_{\ell-i, n}} .
\end{aligned}
$$

We can determine $L_{0}^{W}$ as in Lemma6.8, however, now there is no problem with the normally ordered products because $v^{i} t^{0}$ commutes with $v_{i} t^{0}$. We 
obtain for $\alpha_{0}=0$ and $W=M_{\varphi}\left(R_{a_{1}, a_{2}}\right)$ :

$$
\begin{aligned}
L_{0}^{W} & =\sum_{i=1}^{\ell} \sum_{n=1}^{\infty} x_{i, n}\left(n \partial_{x_{i, n}}-\left(1-\delta_{i, 1}\right) \partial_{x_{i-1, n}}\right) \\
& +\sum_{i=1}^{\ell} \sum_{n=1}^{\infty} x_{\ell+i, n}\left(n \partial_{x_{\ell+i, n}}+\left(1-\delta_{i, 1}\right) \partial_{x_{\ell+i-1, n}}\right) \\
& -\sum_{i=2}^{\ell-1} x_{i, 0} \partial_{x_{i-1,0}}+a_{2} x_{1,0}-a_{1} \partial_{x_{\ell-1,0}}
\end{aligned}
$$

6.5. The case $\operatorname{dim} \mathfrak{h}=2 \ell-1$. Now let $\mathfrak{h}$ be as in Example 6.6. Then $\hat{\mathfrak{h}}_{\varphi}$ is the Lie algebra spanned by a central element $K$ and elements $v_{i} t^{\alpha_{0}+n}$ $(1 \leq i \leq 2 \ell-1, n \in \mathbb{Z})$, with Lie brackets given by:

$$
\left[v_{i} t^{m}, v_{j} t^{k}\right]=m \delta_{m+k, 0} \delta_{i+j, 2 \ell} K+(-1)^{i+1} \delta_{m+k, 0} \delta_{i+j, 2 \ell-1} K .
$$

It follows from (5.2) that for $1 \leq j \leq 2 \ell-1$ :

$$
Y\left(v_{j}, z\right)=\sum_{i=j}^{2 \ell-1} \sum_{m \in \alpha_{0}+\mathbb{Z}}(-1)^{(i-j)(i+j-1) / 2} \zeta^{(i-j)}\left(v_{i} t^{m}\right) z^{-m-1} .
$$

The action of the Virasoro algebra is again determined by (6.8) with $\bar{\omega}=0$. The dual basis $\left\{v^{i}\right\}$ to the basis $\left\{v_{i}\right\}$ is given by $v^{i}=v_{2 \ell-i}$; therefore

$$
L_{k}^{W}=\frac{1}{2} \sum_{i=1}^{2 \ell-1} \sum_{n \in \mathbb{Z}}\left(v_{i} t^{\alpha_{0}+n+k}\right)\left(v_{2 \ell-i} t^{-\alpha_{0}-n}\right), \quad k \neq 0 .
$$

Suppose first that $\alpha_{0}=-1 / 2$; then $\hat{\mathfrak{h}}_{\varphi}^{0}=\mathbb{C} K$ and $R=\mathbb{C}$ with $K=I$. We have:

$$
M_{\varphi}(R) \cong \mathbb{C}\left[x_{j, n}\right]_{1 \leq j \leq 2 \ell-1, n=0,1,2, \ldots},
$$

where for $1 \leq i \leq 2 \ell-1$ and $n=0,1,2, \ldots$,

$$
\begin{aligned}
v_{i} t^{-\frac{1}{2}-n} & =x_{i, n} \\
v_{i} t^{\frac{1}{2}+n} & =\left(\frac{1}{2}+n\right) \partial_{x_{2 \ell-i, n}}+(-1)^{i+1}\left(1-\delta_{i, 2 \ell-1}\right) \partial_{x_{2 \ell-1-i, n}} .
\end{aligned}
$$

As in Lemma 6.8, we find that for $W=M_{\varphi}(R)$,

$$
\begin{aligned}
L_{0}^{W} & =\sum_{i=1}^{2 \ell-1} \sum_{n=0}^{\infty} x_{i, n}\left(\left(\frac{1}{2}+n\right) \partial_{x_{i, n}}+(-1)^{i+1}\left(1-\delta_{i, 1}\right) \partial_{x_{i-1, n}}\right) \\
& +\frac{1}{16}(2 \ell-1) I .
\end{aligned}
$$

Now let $\alpha_{0}=0$. Then $\hat{\mathfrak{h}}_{\varphi}^{0}=\mathfrak{h} t^{0} \oplus \mathbb{C} K$ is a direct sum of a finitedimensional Heisenberg Lie algebra and the central ideal $\operatorname{span}\left\{v_{2 \ell-1} t^{0}\right\}$. The 
following are irreducible $\hat{\mathfrak{h}}_{\varphi}^{0}$-modules with $K=I$ :

$$
R_{a}=\mathbb{C}\left[x_{i, 0}\right]_{1 \leq i \leq \ell-1} \quad(a \in \mathbb{C}),
$$

where

$$
v_{i} t^{0}=x_{i, 0}, \quad v_{\ell-1+i} t^{0}=(-1)^{\ell-i} \partial_{x_{\ell-i, 0}}, \quad v_{2 \ell-1} t^{0}=a I,
$$

for $1 \leq i \leq \ell-1$. Then

$$
M_{\varphi}\left(R_{a}\right) \cong \mathbb{C}\left[x_{i, 0}, x_{j, n}\right]_{1 \leq i \leq \ell-1,1 \leq j \leq 2 \ell-1, n=1,2,3, \ldots},
$$

where for $1 \leq i \leq 2 \ell-1$ and $n=1,2,3, \ldots$,

$$
\begin{aligned}
v_{i} t^{-n} & =x_{i, n}, \\
v_{i} t^{n} & =n \partial_{x_{2 \ell-i, n}}+(-1)^{i+1}\left(1-\delta_{i, 2 \ell-1}\right) \partial_{x_{2 \ell-1-i, n}} .
\end{aligned}
$$

For $W=M_{\varphi}\left(R_{a}\right)$, we have

$$
\begin{aligned}
L_{0}^{W} & =\sum_{i=1}^{2 \ell-1} \sum_{n=1}^{\infty} x_{i, n}\left(n \partial_{x_{i, n}}+(-1)^{i+1}\left(1-\delta_{i, 1}\right) \partial_{x_{i-1, n}}\right) \\
& +\sum_{i=2}^{\ell-1}(-1)^{i+1} x_{i, 0} \partial_{x_{i-1,0}}+\frac{1}{2} \partial_{x_{\ell-1,0}}^{2}+a x_{1,0} .
\end{aligned}
$$

Remark 6.9. After a change of variables, the Virasoro operators (6.10), (6.13) with $\alpha_{0}=a_{1}=a_{2}=a=0$ coincide with those of [EHX, EJX] (see also [DZ1, DZ2]). The detailed correspondence will be discussed elsewhere.

The following special case of Example 6.6 is related to [M].

Example 6.10. Consider an affine Kac-Moody algebra of type $A_{1}^{(1)}$, and let $\mathfrak{h}$ be its Cartan subalgebra. The dual space $\mathfrak{h}^{*}$ has a basis $\left\{\alpha_{1}, \delta, \Lambda_{0}\right\}$ and a nondegenerate symmetric bilinear form $(\cdot \mid \cdot)$ given by:

$$
\left(\alpha_{1} \mid \alpha_{1}\right)=2, \quad\left(\delta \mid \Lambda_{0}\right)=\left(\Lambda_{0} \mid \delta\right)=1,
$$

where all other products of basis vectors are 0 (see [K1, Chapter 6]). The affine Weyl group has an element $\varphi=t_{\alpha_{1}}$, which acts on $\mathfrak{h}^{*}$ by:

$$
\varphi\left(\alpha_{1}\right)=\alpha_{1}-2 \delta, \quad \varphi(\delta)=\delta, \quad \varphi\left(\Lambda_{0}\right)=\Lambda_{0}+\alpha_{1}-\delta .
$$

The bilinear form $(\cdot \mid \cdot)$ is $\varphi$-invariant. Introduce another basis

$$
v_{1}=-\frac{2 \pi \mathrm{i}}{\sqrt{2}} \Lambda_{0}, \quad v_{2}=\frac{\alpha_{1}}{\sqrt{2}}, \quad v_{3}=-\frac{\sqrt{2}}{2 \pi \mathrm{i}} \delta,
$$

so that $\left(v_{i} \mid v_{j}\right)=\delta_{i+j, 4}$. Then $\varphi=e^{-2 \pi \mathrm{i} \mathcal{N}}$ where $\mathcal{N}$ is the linear operator defined by $\mathcal{N}\left(v_{1}\right)=v_{2}, \mathcal{N}\left(v_{2}\right)=-v_{3}$ and $\mathcal{N}\left(v_{3}\right)=0$. 


\section{ACKNOWLEDGEMEnTS}

This paper was motivated by my joint work [BM] with Todor Milanov and our ongoing collaboration. I would like to thank him for many stimulating discussions. I am grateful to Di Yang for sharing his unpublished manuscript [LYZ, and to Dražen Adamović and Antun Milas for discussions on logarithmic CFT. This research was supported in part by a Simons Foundation grant.

\section{REFERENCES}

[AM] D. Adamović and A. Milas, Vertex operator (super)algebras and LCFT. J. Phys. A 46 (2013), no. 49, 494005, 23 pp.

[BK1] B. Bakalov and V.G. Kac, Twisted modules over lattice vertex algebras. In: "Lie theory and its applications in physics V," 3-26, World Sci. Publishing, River Edge, NJ, 2004; math.QA/0402315

[BK2] B. Bakalov and V.G. Kac, Generalized vertex algebras. In: "Lie theory and its applications in physics VI," 3-25, Heron Press, Sofia, 2006; math.QA/0602072.

$[\mathrm{BM}] \quad$ B. Bakalov and T. Milanov, $\mathcal{W}$-constraints for the total descendant potential of a simple singularity. Compositio Math. 149 (2013), 840-888.

[BN] B. Bakalov and N.M. Nikolov, Jacobi identity for vertex algebras in higher dimensions. J. Math. Phys. 47 (2006), no. 5, 053505, 30 pp.

[BPZ] A.A. Belavin, A.M. Polyakov, and A.B. Zamolodchikov, Infinite conformal symmetry in two-dimensional quantum field theory. Nuclear Phys. B 241 (1984), 333-380.

[B] R.E. Borcherds, Vertex algebras, Kac-Moody algebras, and the Monster. Proc. Nat. Acad. Sci. USA 83 (1986), 3068-3071.

[CV] G. Carlet and J. van de Leur, Hirota equations for the extended bigraded Toda hierarchy and the total descendent potential of $\mathbb{C} P^{1}$ orbifolds. J. Phys. A 46 (2013), no. 40, 405205, 16 pp.

[CR] T. Creutzig and D. Ridout, Logarithmic conformal field theory: beyond an introduction. J. Phys. A 46 (2013), no. 49, 494006, 72 pp.

[DK] A. De Sole and V.G. Kac, Finite vs affine $W$-algebras. Japanese J. Math. 1 (2006), 137-261.

[DMS] P. Di Francesco, P. Mathieu, and D. Sénéchal, Conformal field theory. Graduate Texts in Contemporary Physics, Springer-Verlag, New York, 1997.

[DVVV] R. Dijkgraaf, C. Vafa, E. Verlinde, and H. Verlinde, The operator algebra of orbifold models. Comm. Math. Phys. 123 (1989), 485-526.

[DHVW] L. Dixon, J.A. Harvey, C. Vafa, and E. Witten, Strings on orbifolds. Nuclear Phys. B 261 (1985), 678-686.

[D] C. Dong, Twisted modules for vertex algebras associated with even lattices. J. Algebra 165 (1994), 91-112.

[DL] C. Dong and J. Lepowsky, Generalized vertex algebras and relative vertex operators. Progress in Math., 112, Birkhäuser Boston, 1993.

[DZ1] B. Dubrovin and Y. Zhang, Frobenius manifolds and Virasoro constraints. Selecta Math. (N.S.) 5 (1999), 423-466.

[DZ2] B. Dubrovin and Y. Zhang, Virasoro symmetries of the extended Toda hierarchy. Comm. Math. Phys. 250 (2004), 161-193.

[EHX] T. Eguchi, K. Hori, and C.-S. Xiong, Quantum cohomology and Virasoro algebra. Phys. Lett. B 402 (1997), 71-80.

[EJX] T. Eguchi, M. Jinzenji, and C.-S. Xiong, Quantum cohomology and free-field representation. Nucl. Phys. B 510 (1998), 608-622. 
[FFR] A.J. Feingold, I.B. Frenkel, and J.F.X. Ries, Spinor construction of vertex operator algebras, triality, and $E_{8}^{(1)}$. Contemporary Math., 121, Amer. Math. Soc., Providence, RI, 1991.

[FB] E. Frenkel and D. Ben-Zvi, Vertex algebras and algebraic curves. Math. Surveys and Monographs, 88, Amer. Math. Soc., Providence, RI, 2001; 2nd ed., 2004.

[FGM] E. Frenkel, A. Givental, and T. Milanov, Soliton equations, vertex operators, and simple singularities. Funct. Anal. Other Math. 3 (2010), 47-63.

[FK] I.B. Frenkel and V.G. Kac, Basic representations of affine Lie algebras and dual resonance models. Invent. Math. 62 (1980), 23-66.

[FLM] I. B. Frenkel, J. Lepowsky, and A. Meurman, Vertex operator algebras and the Monster. Pure and Appl. Math., 134, Academic Press, Boston, 1988.

[FZ] I.B. Frenkel and Y. Zhu, Vertex operator algebras associated to representations of affine and Virasoro algebras. Duke Math. J. 66 (1992), 123-168.

[Ga] F.R. Gantmacher, The theory of matrices. Vol. 2, AMS Chelsea Pub., Providence, RI, 2000, c1959.

[Go] P. Goddard, Meromorphic conformal field theory. In: "Infinite-dimensional Lie algebras and groups," 556-587, Adv. Ser. Math. Phys., 7, World Sci. Publishing, Teaneck, NJ, 1989.

[HM] R.A. Horn and D.I. Merino, The Jordan canonical forms of complex orthogonal and skew-symmetric matrices. Linear Algebra Appl. 302/303 (1999), 411-421.

$[\mathrm{H}] \quad$ Y.-Z. Huang, Generalized twisted modules associated to general automorphisms of a vertex operator algebra. Comm. Math. Phys. 298 (2010), 265-292.

[K1] V.G. Kac, Infinite-dimensional Lie algebras. 3rd ed., Cambridge Univ. Press, Cambridge, 1990.

[K2] V.G. Kac, Vertex algebras for beginners. University Lecture Series, 10, Amer. Math. Soc., Providence, RI, 1996; 2nd ed., 1998.

[KKLW] V.G. Kac, D.A. Kazhdan, J. Lepowsky, and R.L. Wilson, Realization of the basic representations of the Euclidean Lie algebras. Adv. in Math. 42 (1981), 83-112.

[KP] V.G. Kac and D.H. Peterson, 112 constructions of the basic representation of the loop group of $E_{8}$. In: "Symposium on anomalies, geometry, topology," 276-298, World Sci. Publ., Singapore, 1985.

[KRR] V.G. Kac, A.K. Raina, and N. Rozhkovskaya, Bombay lectures on highest weight representations of infinite dimensional Lie algebras. 2nd ed., Advanced Ser. in Math. Phys., 29. World Sci. Pub. Co. Pte. Ltd., Hackensack, NJ, 2013.

[KT] V.G. Kac and I.T. Todorov, Affine orbifolds and rational conformal field theory extensions of $W_{1+\infty}$. Comm. Math. Phys. 190 (1997), 57-111.

[Le] J. Lepowsky, Calculus of twisted vertex operators. Proc. Nat. Acad. Sci. USA 82 (1985), 8295-8299.

[LL] J. Lepowsky and H. Li, Introduction to vertex operator algebras and their representations. Progress in Math., 227, Birkhäuser Boston, Boston, MA, 2004.

[Li1] H. Li, Local systems of vertex operators, vertex superalgebras and modules. J. Pure Appl. Algebra 109 (1996), 143-195.

[Li2] H. Li, Local systems of twisted vertex operators, vertex operator superalgebras and twisted modules. In: "Moonshine, the Monster, and related topics," 203-236, Contemp. Math., 193, Amer. Math. Soc., Providence, RI, 1996.

[LW] J. Lepowsky and R.L. Wilson, Construction of the affine Lie algebra $A_{1}^{(1)}$. Comm. Math. Phys. 62 (1978), 43-53.

[LYZ] S.-Q. Liu, D. Yang, and Y. Zhang, Semisimple Frobenius manifolds and $\mathcal{W}$ constraints. Unpublished manuscript (2013).

[M] T. Milanov, Hirota quadratic equations for the extended Toda hierarchy. Duke Math. J. 138 (2007), 161-178. 
[MST] T. Milanov, Y. Shen, and H.-H. Tseng, Gromov-Witten theory of Fano orbifold curves, Gamma integral structures and ADE-Toda hierarchies. Preprint (2014), arXiv: 1401.5778 .

[MT] T. Milanov and H.-H. Tseng, The spaces of Laurent polynomials, GromovWitten theory of $\mathbb{P}^{1}$-orbifolds, and integrable hierarchies. J. Reine Angew. Math. 622 (2008), 189-235.

Department of Mathematics, North Carolina State University, Raleigh, NC 27695, USA

E-mail address: bojko_bakalov@ncsu.edu 